\title{
Bolometric Double Beta Decay Experiments: Review and Prospects
}

\author{
Anastasiia Zolotarova ${ }^{+}(\mathbb{D}$ \\ CNRS/IN2P3, IJCLab, Orsay Campus, Université Paris-Saclay, 91405 Orsay, France; \\ anastasiia.zolotarova@ijclab.in2p3.fr \\ † Current address: IRFU, CEA, Université Paris-Saclay, 91191 Gif-sur-Yvette, France.
}

check for

updates

Citation: Zolotarova, A. Bolometric Double Beta Decay Experiments: Review and Prospects. Symmetry 2021, 13, 2255. https://doi.org/ $10.3390 /$ sym 13122255

Academic Editor: Shin'ichi Nojiri

Received: 31 October 2021

Accepted: 16 November 2021

Published: 26 November 2021

Publisher's Note: MDPI stays neutral with regard to jurisdictional claims in published maps and institutional affiliations.

Copyright: (C) 2021 by the author. Licensee MDPI, Basel, Switzerland. This article is an open access article distributed under the terms and conditions of the Creative Commons Attribution (CC BY) license (https:// creativecommons.org/licenses/by/ $4.0 /)$.

\begin{abstract}
This review aims to cover the history and recent developments on cryogenic bolometers for neutrinoless double beta decay $(0 v 2 \beta)$ searches. A $0 v 2 \beta$ decay observation would confirm the total lepton charge non-conservation, which is related to a global $\mathrm{U}(1)_{L C}$ symmetry. This discovery would also provide essential information on neutrino masses and nature, opening the door to new physics beyond the Standard Model. The bolometric technology shows good prospects for future ton-scale experiments that aim to fully investigate the inverted ordering region of neutrino masses. The big advantage of bolometers is the high energy resolution and the possibility of particle identification, as well as various methods of additional background rejection. The CUORE experiment has proved the feasibility of ton-scale cryogenic experiments, setting the most stringent limit on ${ }^{130} \mathrm{Te} 0 v 2 \beta$ decay. Two CUPID demonstrators (CUPID-0 and CUPID-Mo) have set the most stringent limits on ${ }^{82}$ Se and ${ }^{100}$ Mo isotopes, respectively, with compatibly low exposures. Several experiments are developing new methods to improve the background in the region of interest with bolometric detectors. CUPID and AMoRE experiments aim to cover the inverted hierarchy region, using scintillating bolometers with hundreds of $\mathrm{kg}$ of ${ }^{100} \mathrm{Mo}$. We review all of these efforts here, with a focus on the different types of radioactive background and the measures put in place to mitigate them.
\end{abstract}

Keywords: neutrino properties; neutrinoless double beta decay; bolometers; low background techniques

\section{Introduction}

Efforts to obtain a more complete model of fundamental forces and elementary particles have been substantially focused on the determination of neutrino properties in the last years. Nevertheless, significant information on neutrinos is still missing. What is the neutrino nature? Which are the neutrino masses and their ordering [1]? In fact, even though the Standard Model (SM) successfully describes the known elementary particles and their interactions (electromagnetic, weak, and strong), it contains an assumption on neutrino properties - these particles are considered massless in the SM-that has not passed the experimental scrutiny. The results of solar neutrino experiments [2] have shown for decades a significant deficit of the neutrino flux from the sun and a related effect was observed later in the atmospheric neutrino flux. These phenomena were explained by neutrino oscillations, giving strong evidence of physics beyond the SM, as these oscillations-consisting of a periodic interchange between neutrino flavors - can take place only if neutrinos have finite masses. Massive neutrinos can be built in extensions of the SM including a Majorana mass term: this is the most "economical" way to include neutrino masses in the SM. The questions on neutrino nature and masses are a hot topic these days, since the answers to these questions will provide missing elements for an upgraded theory of elementary particles and for cosmology. Numerous experiments are focused on the investigation of neutrino properties and, thanks to the achievement of higher technology levels, the discovery potential of these searches will be very high in the next decade. One of the possible ways to 
get answers to these questions is the research for neutrinoless double beta decay. The detailed description of this process and the connection to neutrino properties are reported in Section 2. Further, we will move to the challenges posed by neutrinoless double beta decay observation in Section 3. As it will be explained in this section, background in the region of interest (ROI) requires particular attention for double beta decay experiments. This review will focus on a specific technology for studies on this process: cryogenic detectors, that have demonstrated to be a powerful and promising technique in the past decades. Section 4 is dedicated to a detailed description of bolometer principles of operation, including a simple thermal model. Different bolometric projects, searching for double beta decay, will be divided according to the technology of background rejection in Section 6, with exception of the CUORE experiment, presented in Section 5. In fact, this experiment has a special position among current bolometric projects, since CUORE has first proven the viability of the bolometric technology on such a big scale and paved the way for the next generation of cryogenic searches for double beta decay.

\section{Neutrinoless Double Beta Decay}

Two main modes of $2 \beta$ decay are usually discussed: two-neutrino double beta decay $(2 v 2 \beta)$ and neutrinoless double beta decay $(0 \nu 2 \beta)$. Both modes are energetically possible for 35 natural isotopes [3]. In the $2 v 2 \beta$ mode, the nucleus emits two antineutrinos and two electrons. This channel is not violating any conservation law, so it is allowed by the Standard Model (SM) and was already observed. The available kinetic energy $Q_{2 \beta}$ is shared among the two electrons and the two antineutrinos, as the energy of the recoiling nucleus is negligible. As the antineutrino fraction of the kinetic energy varies from decay to decay, the sum of the energies of the two electrons has a continuous spectrum. Besides $2 \beta$ decay, there are also other possible processes with the simultaneous change of daughter nucleus charge by two units, such as double positron decay $\left(2 \beta^{+}\right)$, electron capture with positron emission $\left(\varepsilon \beta^{+}\right)$and double electron capture $(2 \varepsilon)$ [3].

Currently, $2 v 2 \beta$ decay was observed in 11 nuclei, and $2 \varepsilon$ capture in 3 nuclei (with some uncertainties for ${ }^{78} \mathrm{Kr}$ results). A recent review [4] summarizes all the measurements of $2 v$ decay processes, with the exception of a very recent update on ${ }^{130} \mathrm{Te}$ half-life [5]. It is worth noting that the results, provided by bolometric experiments, have a very high measurement accuracy of the $2 v 2 \beta$ half-life, reaching $1-2 \%$, while other types of experiments are providing accuracy at the level of $2-10 \%$.

Double beta decay without neutrino emission-proposed for the first time by Furry about 80 years ago [6]-is given by:

$$
(A, Z) \rightarrow(A, Z+2)+2 e^{-}
$$

The main feature of $0 v 2 \beta$ is the violation of the lepton charge by two units. Two matter particles (electrons) are generated without being accompanied by antimatter particles as a counterpart. Since lepton charge conservation is associated with global $U(1)_{L C}$ symmetry, the observation of its violation would have fundamental importance for the new physics beyond the Standard Model. Such a process is possible in many theories beyond the SM with different mechanisms. There is, however, a natural and minimal extension of the SM that is considered as the standard mechanism for $0 \nu 2 \beta$ (often defined as "mass mechanism"). In this framework, the process can be explained by assuming simply the Majorana nature of massive neutrinos in the SM. This means that neutrinos and antineutrinos coincide, like in the case of photons, even though, there is currently no known fermion with this property. The Majorana quantum field can fully describe massive neutrinos, and it causes different physical predictions than in the case of Dirac theory. In particular, $0 v 2 \beta$ would be possible only in the Majorana case. The discovery of Majorana neutrinos would have a great impact on particle physics. If $0 v 2 \beta$ is observed, the neutrino nature will be established, opening the way to the investigation of new physics models. Information on neutrino nature will also lead to a better understanding of the leptogenesis process which may explain the origins of matter-antimatter asymmetry of the Universe. 
The development of $0 v 2 \beta$ experiments is ongoing with the investigation of new detection techniques and data analysis tools. The landscape of $0 v 2 \beta$ search is well described by a nowadays classical plot, showing the dependence of the effective Majorana neutrino mass $m_{\beta \beta}$ - the physics parameter accessible thanks to $0 v 2 \beta$ investigation-on the lightest neutrino mass. In case of the light neutrino exchange mechanism, the $0 v 2 \beta$ decay rate relation with $m_{\beta \beta}$ can be described as follows:

$$
\left(T_{1 / 2}^{0 v}\right)^{-1}=G^{0 v} g_{A}^{4}|N M E|^{2}\left\langle m_{\beta \beta}\right\rangle^{2}
$$

where $G^{0 v}$ is the phase-space factor, whose value is extracted by calculations [7], NME are nuclear matrix elements which are also a product of computation. Currently, NME are the subject of large uncertainties, so there are many efforts that are devoted to their accurate estimation [8,9]. $g_{A}$ is the axial coupling constant in weak interactions. The effective Majorana mass term can be determined through three neutrino eigenstates with masses $m_{1}, m_{2}, m_{3}$ :

$$
m_{\beta \beta}=\left|\sum_{i=1,2,3} U_{e i}^{2} M_{i}\right|
$$

where $U_{e i}$ is the PMNS mixing matrix with two additional Majorana phases [10]. The allowed regions for the effective Majorana mass are constrained by neutrino oscillation parameters and limits on the lightest neutrino mass.

In particular, there are two possible regions mutually exclusive but partially overlapping: the inverted ordering region $\left(m_{3}<m_{1}<m_{2}\right)$ and the direct ordering region $\left(m_{1}<m_{2}<m_{3}\right)$. Currently, double beta decay experiments are just starting the investigation of the region where only the inverted ordering is admitted in Figure 1.

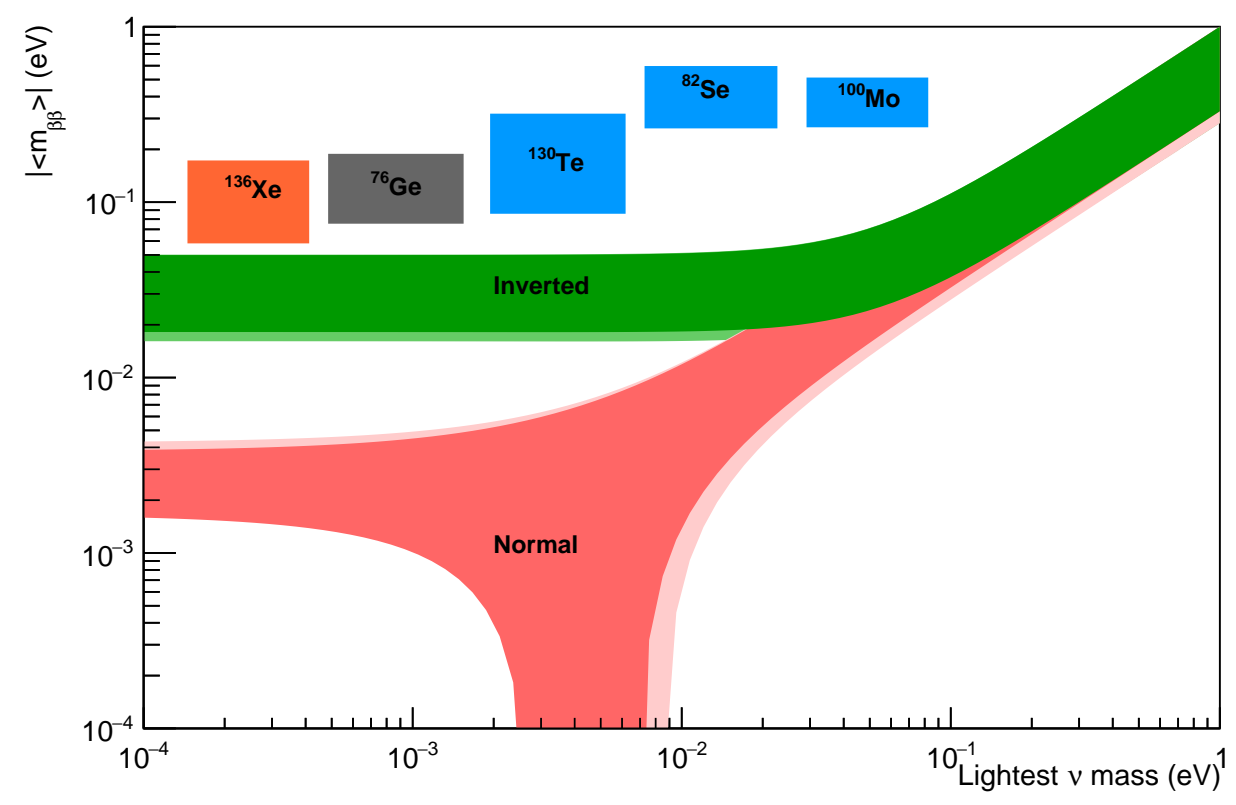

Figure 1. Allowed regions of neutrino masses for inverted (green) and normal (red) ordering. The shaded areas correspond to the $3 \sigma$ regions due to error propagation of the uncertainties on the oscillation parameters. The neutrino oscillation parameters are provided by [11,12]. Creative Commons License CC BY 4.0. The five most stringent limits, obtained experimentally for five different isotopes, are shown with boxes on the plot. All the experiments are covering the full range of lightest neutrino mass, boxes are used for easier reading. ${ }^{136} \mathrm{Xe}$ results are obtained by the KamLAND-Zen experiment using a loaded liquid scintillator detector [13]. Limit on ${ }^{76} \mathrm{Ge} 0 v 2 \beta$ decay is set by the GERDA experiment, operating high-purity germanium diodes [14]. It is worth noting that results, marked by blue color, are provided by bolometric experiments: limit on ${ }^{130} \mathrm{Te}$ is provided by CUORE [15], ${ }^{82} \mathrm{Se}$-by CUPID-0 [16,17], and ${ }^{100} \mathrm{Mo}$-by CUPID-Mo experiment $[18,19]$. 
One can separate the experiments into two groups: calorimetric and tracko-calo detectors. Calorimetric experiments use the "detector = source" approach, which gives an advantage of very high detection efficiency: about $70-90 \%$ up to $\mathrm{FWHM}=0.1 \%$ at $Q_{\beta \beta}$. In tracko-calo experiments, the source and detectors are separated. Now, there is only one collaboration, searching for double beta decay with this approach: the NEMO-3 demonstrator has shown the power of the technology (setting limits on $0 v 2 \beta$ decay for several isotopes [20-23]), and now, the SuperNEMO experiment is in preparation [24]. A thin foil containing the isotope of interest is measured by the tracker, made of wire cells, and the calorimeter, made of scintillating blocks read out by PMTs. This method allows for a full reconstruction of electrons tracks and measurement and identification of background components. However, it has some disadvantages, as low efficiency, energy resolution, and complications with scaling up the detector mass. Calorimetric experiments are more promising for next generations of $0 v 2 \beta$ searches, as the efficiency of the detectors allows to have large exposure within a reasonable time.

Experiments with ultrapure germanium semiconductor diodes, investigating ${ }^{76} \mathrm{Ge}$, have an outstanding energy resolution in the ROI that can be obtained at liquid nitrogen temperature, $\sim 0.2 \%$ FWHM at $2 \mathrm{MeV}$. This kind of detector is well-studied and can be produced in a large amount with masses in the $\mathrm{kg}$ range for a single module. The GERDA experiment [14] recently set a new limit on $0 v 2 \beta$ decay of ${ }^{76} \mathrm{Ge}$ (Table 1 ). Since ${ }^{136} \mathrm{Xe}$ is a noble gas, this isotope can be efficiently studied in liquid scintillator detectors or Time Projection Chambers (TPC). With this kind of technology, the active mass used in the $0 v 2 \beta$ experiment can be bigger than for any other type of detector. The main disadvantage of ${ }^{136}$ Xe experiments is the low energy resolution with respect to other $0 v 2 \beta$ decay searches. The limit obtained by the KamLAND-Zen experiment with a liquid a scintillator technique [13] is the most stringent one up-to-date (Table 1) among all $0 v 2 \beta$ studies with different isotopes, thanks to the advantage of the big exposure. Bolometric experiments, working with dielectric crystals of various compounds, are amongst the most sensitive for several isotopes thanks to a combination of factors: high energy resolution, high efficiency and availability of methods to abate the background [25]. A recent APPEC committee report provides an extensive overview of existing, planned, and proposed technologies for $0 v 2 \beta$, as well as a comparison of technological challenges and discovery potentials for ton-scale experiments [26].

\section{Challenges for Double Beta Decay Searches}

Experimentally, the sensitivity of neutrinoless double beta decays in case of no observation of the effect is given by a limit, corresponding to the half-life of the signal that could be emulated by background fluctuations at a given confidence level. In the general case, this sensitivity is given as follows:

$$
T_{1 / 2}^{0 v 2 \beta}=\frac{\ln (2)}{n_{\sigma}} \frac{N_{A} a \eta \epsilon}{W} f(\Delta E) \sqrt{\frac{M \cdot t}{b \cdot \Delta E}},
$$

where $n_{\sigma}$ is the number of Gaussian standard deviations, $N_{A}$ is the Avogadro constant, $a$ is the isotopic abundance, $\eta$ is the number of the isotope of interest nuclei per molecule of the active mass, $\epsilon$ is the detection efficiency, $W$ is the molecular weight of the active mass, $M$ is the source mass, $t$ is the exposure time, $b$ is the background index (number of counts per mass, time, and energy unit), and $\Delta E$ the energy resolution in the ROI.

Almost all $0 v 2 \beta$ experiments of the next generation are considering the "zero-background approach", where the background index in the ROI should be so small that $b \cdot \Delta E \cdot M \cdot T \ll 1$. In this case, the background fluctuations are replaced by a fixed number that represents the excluded number of counts at a given confidence level when no count is detected in the ROI [27]:

$$
T_{1 / 2}^{0 v 2 \beta}=\frac{\ln (2)}{\ln \left(1-\frac{c . l .}{100}\right)} \frac{N_{A} a \eta \epsilon}{W} \cdot M \cdot t \cdot f(\Delta E)
$$


With this approach, experiments gain a significant improvement in sensitivity with the same exposure.

Theoretically, there are 35 isotopes capable of undergoing $2 \beta$ decay, but only a few of them can be really deployed in ton-scale experiments. A "perfect" choice would be an isotope that has a large natural abundance and/or can be easily concentrated by enrichment, and that can be deployed in large quantity in a background-free experiment with highenergy-resolution and high-efficiency detectors. In Table 1 common isotopes of interest for $0 v 2 \beta$ experiments are shown, as well as lowest half-lives limits, achieved by now. In addition, the calculations of nuclear matrix elements and phase-space factors have to be taken into account: it would be rational to choose the isotope with the highest rate, but again, the possibility of enrichment plays a significant role, as for ton-scale generation of experiments, it may significantly affect the cost of building the detector. In reality, there is no isotope that unites all requirements, thus, experiments have to balance out the isotope choice and the detector design to maximize sensitivity.

Table 1. Natural isotopic abundance and Q-value for common experimentally studied $2 \beta$ emitters. The nuclei are sorted by $\mathrm{Q}$-value in ascending order. The results on $\mathrm{T}_{1 / 2}^{0 v}$ are quoted for the most stringent limits obtained.

\begin{tabular}{|c|c|c|c|c|c|}
\hline Isotope & $\begin{array}{c}\text { Isotopic } \\
\text { Abundance (\%) }\end{array}$ & $Q_{2 \beta}(\mathrm{keV})$ & $\mathrm{T}_{1 / 2}^{0 v}$ Limit, $\mathbf{y r}$ & $\begin{array}{l}\text { Detection } \\
\text { Technology }\end{array}$ & Ref., Year \\
\hline${ }^{48} \mathrm{Ca}$ & 0.2 & 4263 & $6.2 \times 10^{22}$ & $\begin{array}{c}\text { Inorganic } \\
\text { scintillators }\end{array}$ & [28] 2020 \\
\hline${ }^{76} \mathrm{Ge}$ & 7.8 & 2039 & $1.8 \times 10^{26}$ & Semicoductors & [14] 2020 \\
\hline${ }^{82} \mathrm{Se}$ & 9.2 & 2998 & $4.7 \times 10^{24}$ & $\begin{array}{l}\text { Scintillating } \\
\text { bolometers }\end{array}$ & {$[16,17] 2021$} \\
\hline${ }^{96} \mathrm{Zr}$ & 2.8 & 3348 & $9.2 \times 10^{21}$ & $\begin{array}{l}\text { Tracking } \\
\text { calorimeter }\end{array}$ & [29] 2010 \\
\hline${ }^{100} \mathrm{Mo}$ & 9.6 & 3035 & $1.8 \times 10^{24}$ & $\begin{array}{l}\text { Scintillating } \\
\text { bolometers }\end{array}$ & {$[18,19] 2021$} \\
\hline${ }^{116} \mathrm{Cd}$ & 7.6 & 2813 & $1.9 \times 10^{23}$ & $\begin{array}{l}\text { Inorganic } \\
\text { scintillators }\end{array}$ & [30] 2016 \\
\hline${ }^{124} \mathrm{Sn}$ & 5.79 & 2288 & - & - & \\
\hline${ }^{130} \mathrm{Te}$ & 34.1 & 2527 & $3.2 \times 10^{25}$ & $\begin{array}{c}\text { Pure } \\
\text { bolometers }\end{array}$ & [15] 2020 \\
\hline${ }^{136} \mathrm{Xe}$ & 8.9 & 2459 & $1.07 \times 10^{26}$ & $\begin{array}{l}\text { Liquid } \\
\text { scintillator }\end{array}$ & [13] 2016 \\
\hline${ }^{150} \mathrm{Nd}$ & 5.6 & 3371 & $2.0 \times 10^{22}$ & $\begin{array}{l}\text { Tracking } \\
\text { calorimeter }\end{array}$ & [20] 2016 \\
\hline${ }^{238} \mathrm{U}$ & 99.3 & 1150 & $2 \times 10^{21}$ & Geochem. & [31] 1991 \\
\hline
\end{tabular}

\section{Bolometers}

Bolometers are detectors that measure radiation via a temperature change, caused by the interaction of the radiation with the detector's body. A bolometer consists of a single dielectric crystal — the active part of the detector-coupled to a temperature sensor. For double beta decay experiments, the common approach is to use crystal compounds, that contain the isotope of interest, as absorbers. This allows obtaining very high detection efficiency. The detectors are cooled down to very low temperatures, to be able to detect a temperature change after the particle interaction above the noise level.

One of the key features required to build an effective, high-sensitivity bolometric detector is the low heat capacity $C$ of the absorber. The particle interaction in the absorber causes a temperature increase by $\Delta T$, which is proportional to the deposited energy $E$ :

$$
\Delta T=\frac{E}{C}
$$


Currently, the required range of operating temperature is $10-20 \mathrm{mK}$ for most absorbers used in double beta decay experiments.

A simple scheme of a bolometer is shown in Figure 2.

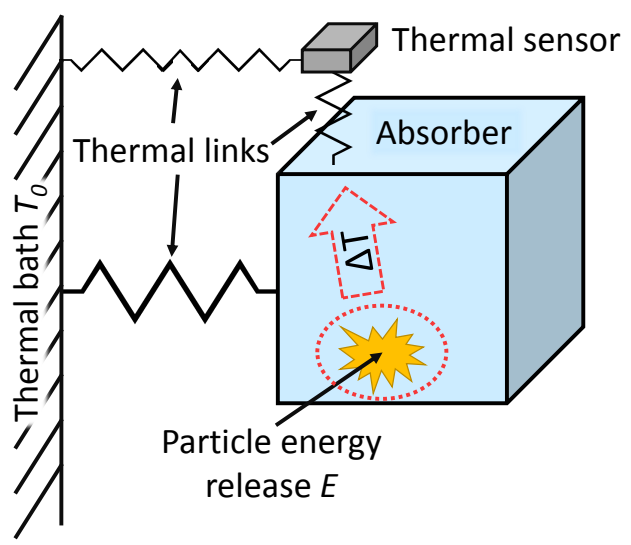

Figure 2. Bolometer scheme. The absorber and the sensor are connected to the heat bath and between them by thermal links.

Thanks to the possibility to embed the isotope of interest in the detector itself, bolometric experiments have very high detection efficiencies.

Such detectors can have an excellent energy resolution in proper conditions-a stable environment, low vibrations, and low electronic noise. The intrinsic limitation to the energy resolution is given by the thermodynamic fluctuations of the internal energy of the detector, due to the random phonon interchanges with the heat bath through the thermal links. Then, the intrinsic RMS noise can be quantified through the fluctuations $\Delta E_{\text {int }}$ of the internal energy: in a simplified derivation, we can assume that the average thermal energy of excitations is $\langle\epsilon\rangle \sim k_{B} T$, and the number of phonons in the absorber $N$ is given by the heat capacity: $N=C(T) / k_{B}$. Then,

$$
\Delta E_{\text {int }}=\sqrt{N} \cdot k_{B} T=\sqrt{k_{B} C(T) T^{2}}
$$

According to this equation, the typical intrinsic resolution of common bolometers, used for $0 v 2 \beta$ is of the order of a few $\mathrm{eV}$. It is worth noting that the actual energy resolutions of such devices are of the order of a few $\mathrm{keV}$ in the ROI. Even though it is possible to show that energy resolutions of the order of magnitude of $\sqrt{k_{B} C(T) T^{2}}$ are expected even when considering the thermistor Johnson noise and the preamplifier noise (if properly optimized), this simple approach is not enough to fully model the energy resolution of a bolometer, since there are other mechanisms to be taken into account, such as energy losses in the detector volume leading to thermalization noise, plus large contributions from external noise sources: microphonics, vibrations, thermal fluctuations, and other parasitic effects.

In the previous discussion, the bolometer was considered as a perfect calorimeter, i.e., a device in which the energy released by an interacting particle is fully converted to heat. Actually, this thermalization process is preceded by a complicate cascade of events. Initially, a hot electron-hole cloud is produced by an ionizing event, as in the case of double beta decay. The relaxation of this electronic system produces excitations of the medium's elastic field, modifying the energy spectrum of the detector's phonon system, which was in thermal equilibrium before the particle interaction. The detector works as a perfect calorimeter only if its time response is slow enough to allow the phonon system to relax on a new equilibrium distribution. Conversely, if the detector response is very fast, the excess non-equilibrium phonons resulting from the electron-hole relaxation can contribute to the signal before they thermalize. However, the microphysics of the detection mechanism is still unexplored and it is difficult to distinguish between these two extreme cases in 
most of the experimental situations. Nevertheless, even when bolometers are not pure calorimeters, their intrinsic energy resolution is better than for conventional detectors, since the typical energy of the primordial excitations (high frequency phonons) is of the order of the Debye energy ( $\sim 10 \mathrm{meV})$, and so very low with respect to conventional detection mechanisms (ionization, gas or scintillation detectors), where it amounts to $\mathrm{eV}^{\prime}$ 's or tens of $\mathrm{eV}^{\prime}$ 's. Detection mediated by non-equilibrium phonons is very attractive, as in principle it contains much information-position of the interaction, discrimination among different types of interacting radiation, and direction of the primary recoil — that is washed out by thermalization [32]. Currently, bolometric detectors found applications in many different fields of fundamental physics, especially in rare event detection [33].

\subsection{Phonon Sensors}

The phonon sensors for bolometric detectors are devices-in strong thermal coupling with the absorber-that convert temperature variations-or excess out-of-equilibrium phonons-into an electrical signal. There are three types of sensors that are used in bolometric double beta decay searches:

- Neutron Transmutation Doped (NTD) Ge thermistors are critically doped semiconductors operated below the metal-insulator transition. They can cover a wide range of temperatures but have relatively slow signals (intrinsically, in the ms time scale). The semiconductor element is usually glued to the absorber [34], typically by twocomponent epoxy, but other solutions are under investigation such as UV-curing glue. They are easily reproducible on large scales. NTDs do not require electronics at cold stages-although this can help in some situations. The signal consists of a voltage change, and only two wires per sensor are needed for bias and read out. The typical NTD response is $\sim 10 \mathrm{~ms}$ for the rise time and hundreds of ms for the decay time in large mass crystals.

- Transition Edge Sensors (TES) consist of superconductive films operated in a narrow neighborhood around the transition temperature $T_{c}$, where the resistance of the sensor changes abruptly with small variations of the temperature. TESs can be directly evaporated on the absorber surface, providing a much faster response and giving the sensitivity to athermal phonons rather than thermal in the case of gluing [35]. Due to the low impedance, optimal readout requires the use of superconducting quantum interference devices (SQUIDs), which may be a complication, especially in the case of hundreds or thousands of channels.

- Metallic Magnetic Calorimeters (MMC) use materials with magnetization-temperature dependence. They have high energy resolution and fast signals, but they also require a readout system based on SQUIDs [36].

\subsection{Cryostats}

An essential component of the bolometric experiments is the cryogenic facility itself, which shall provide a possibility to measure arrays of bolometric detectors in stable conditions for years. The use of dilution refrigerators (DR) can provide cooling down of the samples and maintain stable conditions at $\mathrm{T} \approx 10-20 \mathrm{mK}$.

The development of cryogenic facilities over the years has shown huge progress in stability, size, and efficiency of use. We observed a transition over the last years from cryostats that used cryogenic liquids to pulse-tube cryostats capable of pre-cooling to $\approx 4 \mathrm{~K}$ temperatures with a thermal machine. This transition gives a big advantage in terms of budget - no need to buy big amounts of liquid helium, just using a closed-cycle system. The drawback of the pulse-tube cryostats is the increase of the vibration level, as the pulse tube is a source of vibrations at low frequencies. Significant progress was made to improve the vibrations level and decoupling of the detectors [37-39]. The CUORE experiment has proven that such a complex facility can be operated in stable conditions for a ton-scale detector. This demonstration has opened the door for the next generation of bolometric experiments. 


\subsection{Bolometers for $0 v 2 \beta$ Searches}

Bolometric experiments propose detectors with high energy resolution $\left(\approx 0.2 \%\right.$ at $\left.Q_{2 \beta}\right)$, but they are limited by:

(a) Crystal compounds that can contain the isotopes of interest-it should be possible to embed such isotope into a dielectric single crystal, and such crystals should be grown in large quantities with good reproducibility;

(b) Size of a cryostat-currently, the largest cryostat is the CUORE one, which is able to host around 1 cubic meter of detectors. Scaling beyond this size was never done before, and requires dedicated technological research;

(c) Available technologies for background reduction.

Experiments and working prototypes based on bolometers-at very different maturity levels - have been performed with the aim of investigating the six nuclides ${ }^{48} \mathrm{Ca}$, ${ }^{100} \mathrm{Mo},{ }^{82} \mathrm{Se},{ }^{116} \mathrm{Cd},{ }^{130} \mathrm{Te}$ and ${ }^{124} \mathrm{Sn}$. Competitive experiments have been performed and are planned for ${ }^{130} \mathrm{Te}$ (CUORE experiment with natural $\mathrm{TeO}_{2}-$ Section 5, an R\&D with Cherenkov light tagging for $\alpha$ rejection-Section 6.2 .6$),{ }^{100} \mathrm{Mo}$ (this isotope has a high priority: several experiments are working on its investigation on different scales: CUPID-Mo demonstrator, Section 6.2.2, has finished its data taking and technology is adopted for CUPID, the future ton-scale detector, Section 6.2.7. AMoRE experiment is ongoing, scaling to $\sim$ hundreds kg detector, Section 6.2.3. BINGO, Section 6.1.1 and CROSS, Section 6.3.1 experiments are investigating additional tools for background reduction), and ${ }^{82} \mathrm{Se}$ (CUPID-0 demonstrator, Section 6.2.1). R\&D is ongoing on ${ }^{48} \mathrm{Ca}$ (described in Section 6.2.4) and ${ }^{124} \mathrm{Sn}$. ${ }^{116} \mathrm{Cd}$ was studied with few prototypes, described in Section 6.2.5. (The case of ${ }^{76} \mathrm{Ge}$ is special: highly-performing bolometers can be fabricated using single Ge crystals, but they are not employed in $0 v 2 \beta$ search as the technique based on large-volume Ge diodes is more competitive in terms of energy resolution and background control).

All the above-mentioned isotopes and the related bolometric experimental efforts will be discussed in the next sections-following an approach privileging the techniques for the control of the background - with the exception of the less mature ${ }^{124} \mathrm{Sn}$ case, that will be shortly treated here. The INdia-based TIN Detector (TIN.TIN) is an experiment aiming at studying ${ }^{124} \mathrm{Sn} 0 v 2 \beta$ decay [40]. The detector will be housed at the upcoming underground facility in the India-based Neutrino Observatory (INO). The project is in the R\&D phase, and reasonable performance of tin bolometers was demonstrated only with small-scale samples. An array of tin microcalorimeters with TES sensors was measured [41], achieving $22 \mathrm{keV}$ FWHM resolution at a $97.43 \mathrm{keV}$ line. However, the detector size has to be scaled up a lot for double beta searches, and fabricating a large size superconducting bolometer appears to be a challenge. It was observed that the thermalization time in superconductors can be large compared to insulating bolometers. The experiment has planned, as a next step, to build detector with a 27 element array of $3 \times 3 \times 3 \mathrm{~cm}^{3}$ tin crystals [42]. However, additional complications are arising due to tin pest-currently, it is a major concern for TIN.TIN detectors development, and various alloys have been tested [43]. If the chosen tin alloys will meet the requirements for cryogenic operation, one has to confirm that radiopurity levels are sufficient for a sensitive $0 v 2 \beta$ decay experiment.

\section{CUORE Experiment: A Saga of $\mathrm{TeO}_{2}$ Bolometers}

The Cryogenic Underground Observatory for Rare Events (CUORE) is the first bolometric experiment that has confirmed the viability of cryogenic detectors technology on a ton scale. The cryostat and its infrastructure is located in the underground Gran Sasso National Laboratory (Italy). The detector consists of an array of $988 \mathrm{TeO}_{2}$ crystals arranged in a compact cylindrical structure of 19 towers, for a total active mass of $742 \mathrm{~kg}$, and $206 \mathrm{~kg}$ of ${ }^{130} \mathrm{Te}$. Thanks to the high natural abundance of ${ }^{130} \mathrm{Te}(34 \%)$, isotopic enrichment was not required in CUORE. The average energy resolution at the ROI is 7.7(5) $\mathrm{keV}$. The current limit on $\mathrm{T}_{1 / 2}$ is $3.2 \times 10^{25} \mathrm{yr}$, which corresponds to an upper limit on the effective Majorana mass at 75-350 $\mathrm{meV}[15]$. 
The development of $\mathrm{TeO}_{2}$ bolometers, which led to the CUORE experiment, started in the early 1990s. The first prototype was a 6-g crystal, and starting from that, $\mathrm{TeO}_{2}$ bolometers were progressing fast with an increase in size, performance, and modularity. The CUORICINO experiment has demonstrated the viability of cryogenic double beta decay experiments on a mid scale [44], leading to the construction of CUORE-0, a single tower prototype [45].

CUORE clearly demonstrated the power of the bolometric technology, being competitive at the ton scale level and being currently the third most sensitive $0 v 2 \beta$ experiment worldwide.

A recent review of CUORE development and achievements describes extensively the path made to obtain these results [46].

Not only the CUORE experiment provided the most stringent limit on the $0 v 2 \beta$ decay of ${ }^{130} \mathrm{Te}$ and one of the best worldwide on the effective Majorana mass, but it also produced an essential outcome for future bolometric experiments: the CUORE background model, which clearly shows the impact of the various components on the background level in the ROI [47].

The CUORE background model teaches two main lessons, which guide the choices to be taken in any future bolometric experiments:

1. In pure bolometers, without additional active tools for background rejection, the $\alpha$ background coming from surface contamination-either of the crystals themselves or of the material surrounding them-dominate at the level of $10^{-2}$ counts $/ \mathrm{keV} / \mathrm{kg} / \mathrm{yr}$, see Figure 3. This contribution is flat in the energy spectrum over a large range, as the $\alpha$ energy is degraded due to a partial absorption in passive materials. This range covers the ROI not only of ${ }^{130} \mathrm{Te}$ but also of all the most promising candidates.

2. The subdominant contribution is due to external $\gamma$ background, at the level of $10^{-3}$ counts $/ \mathrm{keV} / \mathrm{kg} / \mathrm{yr}$. This is specific of candidates-as ${ }^{130} \mathrm{Te}$-with a ROI below $\sim 2.6 \mathrm{MeV}$, where the bulk of the $\gamma$ natural radioactivity is located. Above this value, the $\gamma$ background level drops by at least an order of magnitude. This contribution can in principle be reduced by a stricter selection of the materials for the cryostat and the shielding.

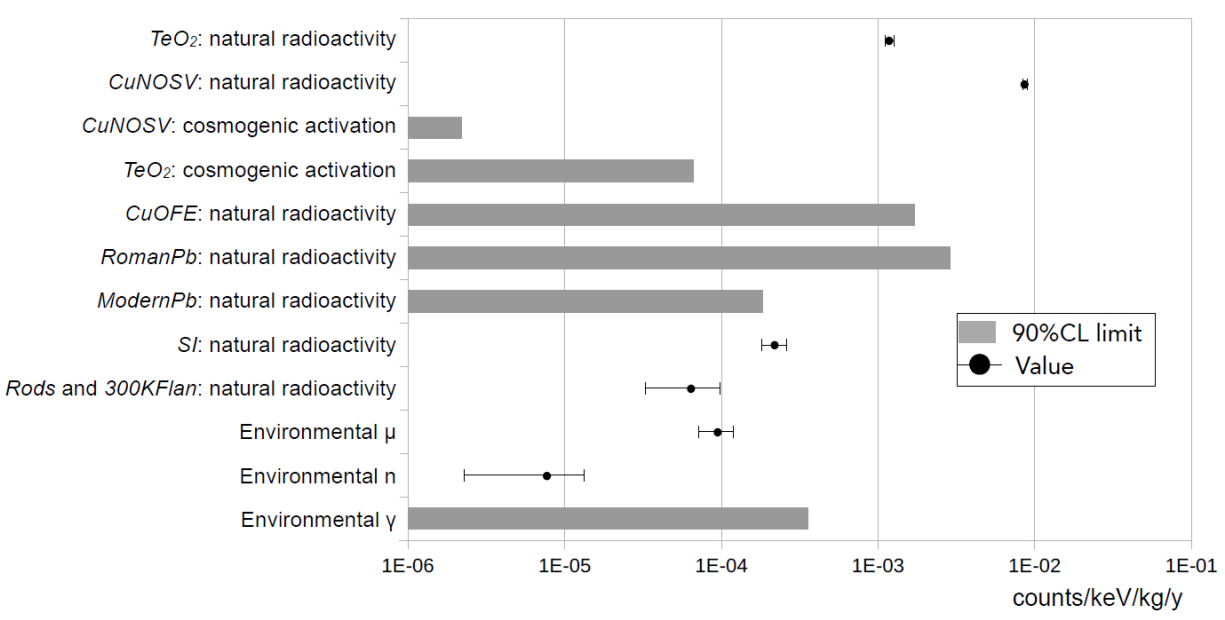

Figure 3. Histogram representing the main background indices expected for the various components of CUORE. The gray bars indicate 90\% C.L. upper limits while the dots (with $1 \sigma$ uncertainties) indicate derived values. Only statistical uncertainties are indicated. The figure is reprinted with permission from [47]. Creative Commons License CC BY 4.0.

The CUORE detector was composed of "classical" bolometric materials-copper, PTFE, epoxy glue. We do not expect that these materials can be fully replaced or purified much more for future bolometric experiments. In terms of surface cleaning, an arsenal of costly and time-consuming operations was deployed in the preparation of the CUORE 
detector. It is imprudent to count on a more effective surface treatment in the design of future experiments. Therefore, the improvements must rely on the isotope choice and on new concepts in the detector technology aiming at background rejection.

In conclusion, CUORE shows us that the background index in the ROI depends first on the location of the ROI itself [47]. It is critical if the transition energy is below $2.6 \mathrm{MeV}$, as in the case of ${ }^{130} \mathrm{Te}$. Here, we see a relevant contribution of the $\gamma$ background, which could be controlled with a novel technique proposed in BINGO (Section 6.1.1).

If we move to isotopes with transition energies above $2.6 \mathrm{MeV}$ (for instance, ${ }^{82} \mathrm{Se}$ and ${ }^{100} \mathrm{Mo}$ have Q-values around $3 \mathrm{MeV}$ ), we still have to cope with the major contribution from surface $\alpha$ events, which is typically at the level of $10^{-2}$ counts $/ \mathrm{keV} / \mathrm{kg} / \mathrm{yr}$. The rejection of $\alpha^{\prime}$ s (Section 6.2) — either with the use of scintillating bolometers or with surface-sensitive bolometers as in CROSS (Section 6.3.1) - and the simultaneous use of an isotope with a Q-value above $2.6 \mathrm{MeV}$ brings the background index in the region $10^{-3}-10^{-4}$ counts $/ \mathrm{keV} / \mathrm{kg} / \mathrm{yr}$, as demonstrated in CUPID-0 (Section 6.2.1) and CUPID-Mo (Section 6.2.2) and predicted in CUPID (Section 6.2.7) and AMoRE (Section 6.2.3). In particular, CUPID can be considered as the direct follow-up of CUORE as it aims at using the same cryogenic infrastructure, although it adopts the CUPID-Mo technology.

Further improvements of CUPID are already under discussion at the R\&D level with the aim to reach a background index of the order of or below $10^{-5}$ counts $/ \mathrm{keV} / \mathrm{kg} / \mathrm{yr}$. This goal requires the discrimination also of the $\beta$ background (Section 6.3), especially from surface contamination (possible with CROSS and BINGO), and the control of the background induced by the random coincidences of $2 v 2 \beta$ decay events (Section 6.4), if ${ }^{100}$ Mo-enriched bolometers are used.

\section{Approaches to Reduce Different Background Contributions}

Background is the keyword in $0 v 2 \beta$ decay experiments [48]. The bolometric technology provides several possibilities to control different forms of background [49]. We will discuss here these approaches-grouped according to the background source-and the experiments or the R\&D's in which they are implemented. We will omit the description of methods to reject residual cosmic muon and neutron background in underground laboratories-based essentially on active vetos located externally to the detector setup-as they are not specific for the bolometric technology.

\section{1. $\gamma$ Background}

The detectors in double beta decay experiments are always installed underground and heavily shielded to reduce external backgrounds. Still, after the reduction, the main sources of contamination are the detector construction materials, that can be a close background source. In addition, the experience of CUORE shows that external $\gamma$ background can still play a relevant role. Omnipresent radioisotopes are from ${ }^{238} \mathrm{U}$ and ${ }^{232} \mathrm{Th}$ natural radioactivity chains. ${ }^{208} \mathrm{Tl}$ is the isotope with the highest energy $\gamma$ line from these chains, at $2615 \mathrm{keV}$. As discussed above, choosing $0 v 2 \beta$ isotope with $Q_{2 \beta}$ above this value gives a significant advantage for background in the ROI.

The reduction of contamination of detectors materials is achieved through the production of radiopure materials or purification with specific techniques. Radiopurity screening of all detector components is always required. The screening results are often shared and aid the process of materials selection for future experiments.

Exploiting the fact that bolometric detectors are usually tight-packed arrays, the application of anti-coincidence analysis allows to exploit the high granularity to reject multi-site events, such as multi-Compton interactions induced by $\gamma$-rays and muons crossing more than one detector.

\subsubsection{BINGO}

BINGO is a recent project focused on building a demonstrator with an efficient $\gamma$ background rejection. This experiment will use $\mathrm{TeO}_{2}$ and $\mathrm{Li}_{2} \mathrm{MoO}_{4}$ crystals for the demon- 
strator, going along with the CUPID experiment. The key points of background reduction will be:

- The use of an active shield, based on $\mathrm{ZnWO}_{4}$ or $\mathrm{BGO}$ scintillators with bolometric readout, to suppress the external $\gamma$ background;

- The increase of the light detector sensitivity thanks to Neganov-Luke amplification;

- A new type of detector assembly that will reduce the total surface radioactivity contribution.

The first point is particularly relevant in the discussion on $\gamma$ background. For the first time in a large array of macrobolometers, an active inner shield will be installed directly in the experimental space in the $\mathrm{mK}$ region to reduce the contribution coming from external $\gamma$ radioactivity. This is indispensable in the more difficult case of ${ }^{130} \mathrm{Te}$, but relevant in general if a background index below $10^{-5}$ counts $/ \mathrm{keV} / \mathrm{kg} / \mathrm{yr}$ is targeted.

The internal shield proposed in BINGO is an almost hermetic arrangement of scintillating bars, forming a barrel all around the arrays of crystals and placed nearby the bolometers. Each bar will be a single $\mathrm{ZnWO}_{4}$ crystal. This material is chosen because of its attractive features: high density $\left(7.8 \mathrm{~g} / \mathrm{cm}^{3}\right)$; high average $\mathrm{Z}(\sim 51)$; possibility to grow large crystals; high scintillation yield, of the order of at least 9300 photons $/ \mathrm{MeV}$ at room temperatures and largely improvable at low temperatures; long light attenuation length $(\sim 20-30 \mathrm{~cm})$ at the peak emission wavelength $(480 \mathrm{~nm})$; impressive achievable radiopurity $\left(<0.17 \mu \mathrm{Bq} / \mathrm{kg}\right.$ in $\left.{ }^{228} \mathrm{Th}\right)$.

Each bar will be read out by two Neganov-Luke Ge-based light detectors at its extremities. It is worth remarking that the $\mathrm{ZnWO}_{4}$ crystals will not be operated as bolometers, but as pure scintillators (with a major simplification of the assembly). Only light detection will be performed bolometrically, as this choice is quite convenient in a mK environment. Preliminary evaluations point at an active-shield thickness of about $10 \mathrm{~cm}$.

Once that the concept is validated by BINGO, the objective in next-generation experiments will be the design and the installation of an active shield capable of reducing the background observed in CUORE by at least two orders of magnitude [50].

\section{2. $\alpha$ Background}

$\alpha$ contamination can be divided into two main subcategories: bulk and surface. Bulk contamination from nuclei belonging to the natural radioactive chains is mostly harmless in terms of $\alpha$ particles: energy is far above the ROI (with the exception of $\mathrm{Ca}^{48}$ ), and all the energy of decay is absorbed. In case of surface contamination, the energy can be absorbed only partially, and $\alpha$ events would contribute to the background in the ROI. Of course, both bulk/surface contamination should be minimized. The minimization of bulk contamination is achieved by powder purification and double crystallization [51] and purification or appropriate selection of detector surrounding materials.

Surface contamination can be reduced by special procedures for surface cleaning (like it was done in CUORE [52]), performing of assembly and detectors installation in clean-room environments, and glove boxes to reduce the Rn contamination [53].

Further improvement of the background can be achieved by particle identification and active $\alpha$ rejection. A well-developed method for $\alpha$ identification is the use of scintillating bolometers: the crystal absorber, depending on the compound, can scintillate. An auxiliary bolometer, sensitive in the light energy range, can be coupled to the scintillating crystal in order to measure a light signal simultaneously. The amplitude of the light signal from $\alpha$ particles is usually lower than the one from a $\gamma / \beta$ interaction with the same energy and this difference allows to get separation between two particle bands.

There are several experiments that have already shown efficient rejection of $\alpha$ particles, using the technique of scintillating bolometers, and many crystals tested for rare decay searches [54]. 


\subsubsection{CUPID-0: $\mathrm{Zn}^{82}$ Se Demonstrator}

CUPID-0 is the first large array of scintillating bolometers, using $\mathrm{Zn}^{82} \mathrm{Se}$ crystals coupled to germanium light detectors and NTD sensors for both heat and light channels.

The detector consisted of 24 enriched $\mathrm{Zn}^{82} \mathrm{Se}+2$ natural abundance bolometers for a total ${ }^{82} \mathrm{Se}$ mass of $5.28 \mathrm{~kg}$ and it has been taking data in the underground Gran Sasso National Laboratory (Italy) till the end of 2018 (see Figure 4). CUPID-0 has demonstrated the potential of the scintillating bolometer technology and has set the most stringent limit on the half-life of ${ }^{82} \mathrm{Se} 0 v 2 \beta: \mathrm{T}_{1 / 2}^{0 v}>4.7 \times 10^{24} \mathrm{yr}(90 \% \mathrm{C}$. L.), corresponding to $\mathrm{m}_{\beta \beta}<276-570 \mathrm{meV}$, depending on the nuclear matrix element calculations $[17,55]$. The energy resolution at the Q-value of ${ }^{82} \mathrm{Se}$ is $20.05 \pm 0.34 \mathrm{keV} F W H M$, and the background index in ROI was $3.5_{-0.9}^{+1.0} \times 10^{-3}$ counts $/ \mathrm{keV} / \mathrm{kg} / \mathrm{yr}$ - at that moment, it was the lowest background achieved by $0 v 2 \beta$ bolometric experiments [16].
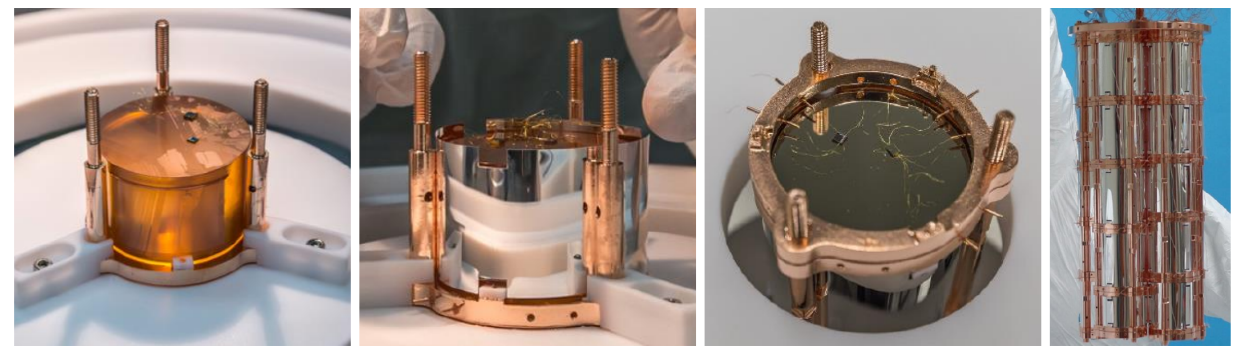

Figure 4. Pictures of the CUPID-0 detector in process of assembly. From left to right: a ZnSe crystal, the same crystal surrounded by the reflecting foil, the Ge light detector mounted on top, the CUPID-0 array of 26 scintillating calorimeters. The figure is reprinted with permission from [56].

Thanks to high signal-to-noise ratio and good energy resolution, the most precise value up to date on ${ }^{82} \mathrm{Se} 2 v 2 \beta$ decay was obtained: $T_{1 / 2}^{2 v}=8.6_{-0.1}^{+0.2} \times 10^{19} \mathrm{yr}$ [57]. In the case of $0 v 2 \beta$ observation in other isotopes, this technology can be used in the future at a bigger scale seeking a possible confirmation in ${ }^{82} \mathrm{Se}$.

In $\mathrm{Zn}^{82}$ Se detectors, the $\alpha$ light yield is higher than the one of $\gamma / \beta$, which is not the typical behavior for scintillators. In addition, a fraction of the $\alpha$ events shows a reduced light yield that leads them to leak in the $\gamma / \beta$ band in a heat-light scatter plot. Due to this feature, CUPID-0 analysis used not only the amplitude of the light signal but mostly the pulse shape of the light detector signal for better discrimination, as shown in Figure 5.
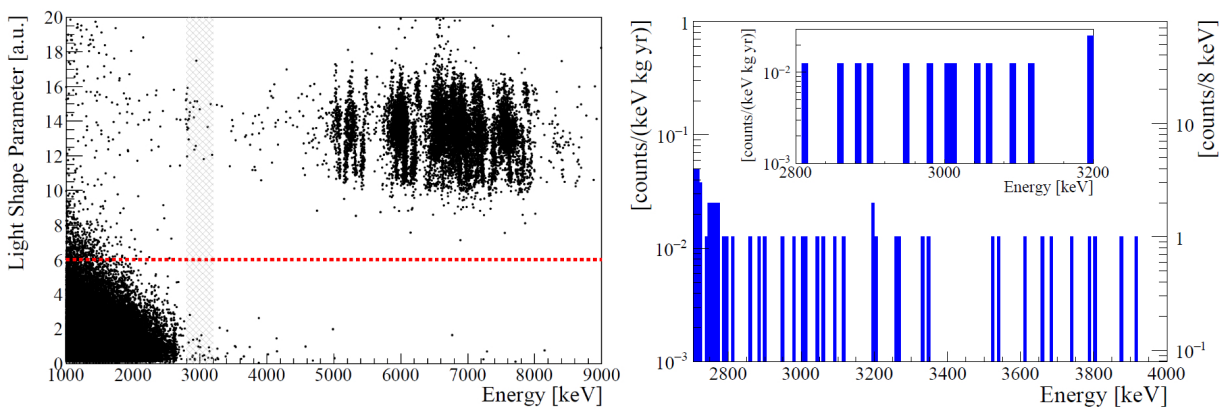

Figure 5. Left: Single-hit events in the Light Shape Parameter-Energy plane. The red dotted line indicates the acceptance threshold. The shaded vertical band represents the analysis window used for the background evaluation at $\mathrm{Q}_{2} \beta$. This plot clearly demonstrates the efficiency of $\alpha$ background rejection. The figure is reprinted with permission from [58]. Right: Background measured by CUPID0 (exposure of $9.95 \mathrm{~kg} \times \mathrm{yr}$ of $\mathrm{Zn}^{82}$ Se crystals). Inset: energy ROI for the $0 v 2 \beta$ search: from 2.8 to 3.2 MeV, a symmetric energy region around the Q-value of ${ }^{82} \mathrm{Se}(2997.9 \pm 0.3 \mathrm{keV})$. The figure is reprinted with permission from [16].

The CUPID-0 experiment has led to many significant physics results-in particular, it confirmed the single state dominance at the level of 5.5 $\sigma$ [55] in two-neutrino double beta 
decay of ${ }^{82} \mathrm{Se}$, performed a search for Lorentz violation for the first time with scintillating bolometers [59], and set limits on $0 v 2 \beta$ for the isotopes ${ }^{64} \mathrm{Zn}$ and ${ }^{70} \mathrm{Zn}[60]$.

\subsubsection{CUPID-Mo: $\mathrm{Li}_{2}{ }^{100} \mathrm{MoO}_{4}$ Demonstrator}

Another demonstrator for CUPID with scintillating bolometers was investigating the ${ }^{100} \mathrm{Mo}$ isotope with $\mathrm{Li}_{2}{ }^{100} \mathrm{MoO}_{4}$ crystals and germanium light detectors, coupled to NTD sensors. The detector, consisting of 20 modules (an example of one module is shown in Figure 6) was installed in the Modane underground laboratory, sharing the cryostat with the EDELWEISS experiment [61].

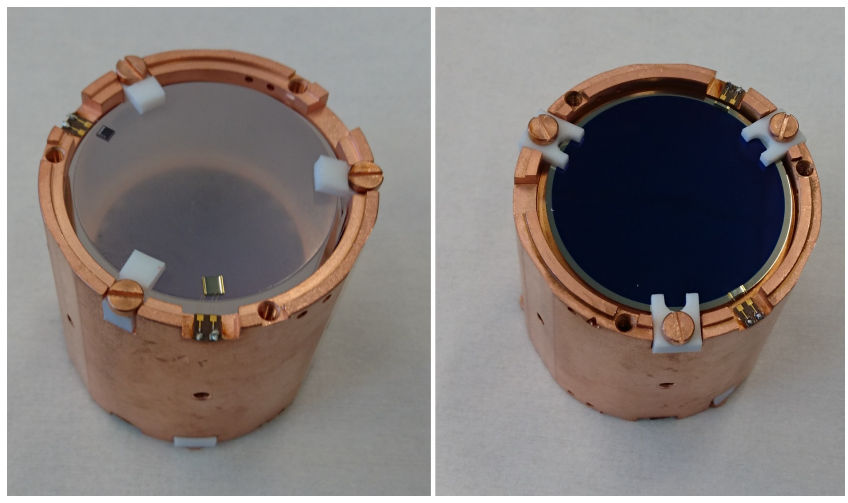

Figure 6. An assembled CUPID-Mo module. Left: view from the top, the $\mathrm{Li}_{2}{ }^{100} \mathrm{MoO}_{4}$ crystal is fixed with help of white teflon parts, NTD and heater are glued on the top surface of the crystal. Right: view from the bottom, germanium LD is also fixed with teflon parts. The dark blue internal circle is the antireflective $\mathrm{SiO}$ coating. An NTD is glued close to the edge of the light detector. The photos are reprinted with permission from [62]. Creative Commons License CC BY 4.0.

The energy resolution-over the all-channel-summed spectrum-was of $5.3 \mathrm{keV}$ (6.5 keV) FWHM at $2615 \mathrm{keV}$ with Th calibration (background) measurement. The measured light yield for $\gamma / \beta$ events $(0.6-0.9 \mathrm{keV} / \mathrm{MeV})$ is compatibly low with respect to other common scintillating bolometers, but thanks to the high, achieved baseline resolution of bolometric Ge light detectors (146 eV FWHM), full $\alpha$ to $\gamma / \beta$ separation is obtained. The $\mathrm{Li}_{2}{ }^{100} \mathrm{MoO}_{4}$ crystals also demonstrated a high level of radiopurity: $\leq 3 \mu \mathrm{Bq} / \mathrm{kg}$ of ${ }^{226} \mathrm{Ra}$ and $\leq 2 \mu \mathrm{Bq} / \mathrm{kg}$ of ${ }^{232} \mathrm{Th}[18,19]$. Similarly to CUPID-0, with very high signal-tonoise ratio, a precise measurement of ${ }^{100}$ Mo $2 v 2 \beta$ decay half-life was obtained: $T_{1 / 2}^{2 v}=$ $\left[7.12_{-0.14}^{+0.18}(\right.$ stat $) \pm 0.10($ syst $\left.)\right] \times 10^{18}$ yr [63].

Recently, the limit on $0 v 2 \beta$ was published, confirming the success of the technology (see Figure 7, showing the efficiency of $\alpha$ discrimination and the final spectrum). This result surpassed the previous best limit (set by NEMO-3, tracko-calorimetric experiment, with exposure of $35 \mathrm{~kg} \times \mathrm{yr}$ [22] on $0 v 2 \beta$ of ${ }^{100} \mathrm{Mo}$ ) and set up a new one at $\mathrm{T}_{1 / 2}^{0 v}>1.8 \times 10^{24}$ year ( $90 \%$ confidence interval), which corresponds to $\mathrm{m}_{\beta \beta}<280-490 \mathrm{meV}$, with an exposure of only $2.71 \mathrm{~kg} \times$ year $[18,19]$. This is the fourth most stringent limit worldwide among all isotopes/experiments. ${ }^{100}$ Mo-based experiments gain an advantage thanks to favorable predictions on $0 v 2 \beta$ decay half-life, extracted from nuclear matrix element calculations [8,9]. This result, together with CUPID-0 demonstrators, shows the advantage and perspective of bolometric technology for double beta decay searches. The $\mathrm{Li}_{2}{ }^{100} \mathrm{MoO}_{4}$-based detectors were chosen as the baseline for CUPID—the ton-scale experiment that will use the CUORE infrastructure. 

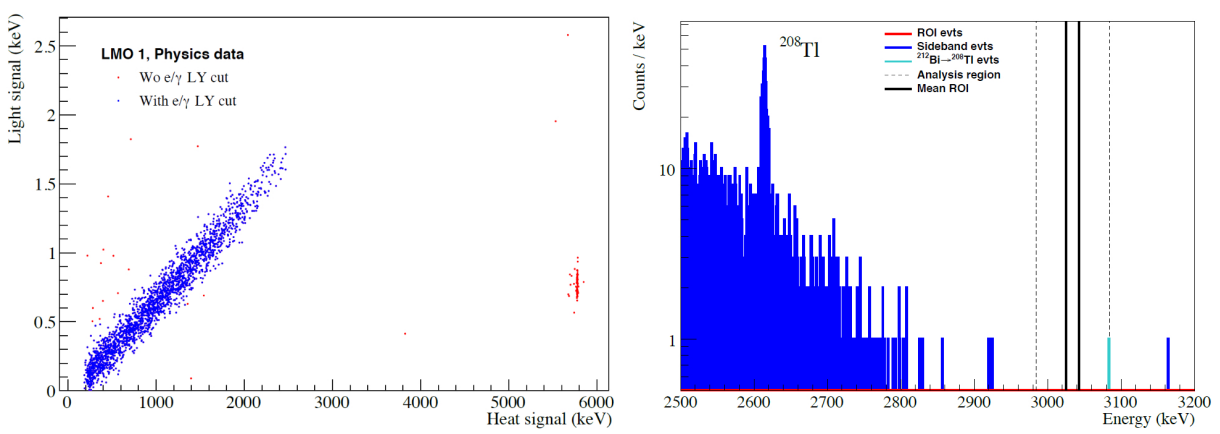

Figure 7. Left: Light yield versus heat signal scatter-plot using 11 days of physics data from one detector. The figure is reprinted with permission from [62]. Creative Commons License CC BY 4.0. Right: Physics spectrum for $2.16 \mathrm{~kg} \times \mathrm{yr}$ of data after unblinding. No event is observed in the detector and dataset-based ROI. The figure is reprinted with permission from [18].

\subsubsection{AMoRE Experiment}

The AMoRE experiment is exploiting the scintillating-bolometer technology with the use of molybdates containing crystal scintillators and germanium light detectors, equipped with MMC sensors.

The AMoRE experiment includes several phases of measurements (see also Figure 8 for the configuration of the detectors in each phase):

- $\quad$ AMoRE-pilot: R\&D phase, measurements of $6{ }^{48 d e p l} \mathrm{Ca}^{100} \mathrm{MoO}_{4}$ crystals $(0.9 \mathrm{~kg}$ of $\left.{ }^{100} \mathrm{Mo}\right)$, is finished;

- $\quad$ AMoRE-I, $13{ }^{48 d e p l} \mathrm{Ca}^{100} \mathrm{MoO}_{4}$ and $6 \mathrm{Li}_{2}{ }^{100} \mathrm{MoO}_{4}$ crystals $(6 \mathrm{~kg})$ is ongoing;

- $\quad$ AMoRE-II is the final phase, planned to have $\sim 100 \mathrm{~kg}$ of ${ }^{100} \mathrm{Mo}$.
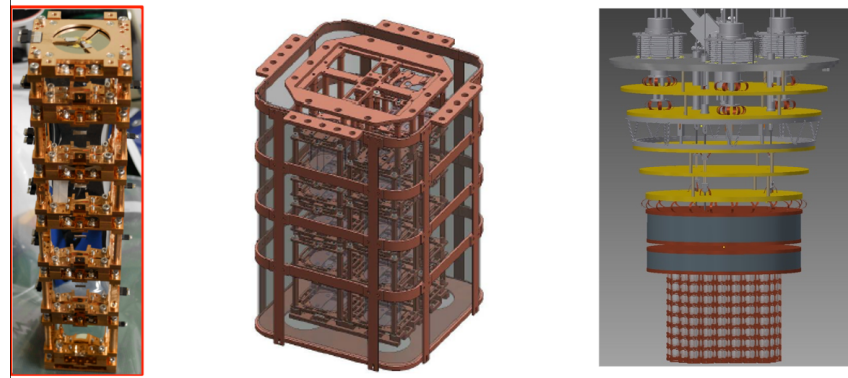

Figure 8. Left: assembled tower of AMoRE-Pilot with six $\mathrm{CaMoO}_{4}$ crystals ( $\sim 1.9 \mathrm{~kg}$ in total). Middle: schematic design of the AMoRE-I configuration with $13 \mathrm{CaMoO}_{4}$ crystals and $5 \mathrm{Li}_{2} \mathrm{MoO}_{4}$ crystals ( $\sim 6 \mathrm{~kg}$ in total). Right: design of the AMoRE-II detector with $\sim 400$ molybdate crystals ( $\sim 200 \mathrm{~kg}$ in total) beneath lead shields and cold plates. The figure is reprinted with permission from [64]. Creative Commons License CC BY 4.0.

The average energy resolution of ${ }^{48 d e p l} \mathrm{Ca}^{100} \mathrm{MoO}_{4}$ crystals at ROI was $14.4 \mathrm{keV}$. The first result from AMoRE-Pilot from an energy spectrum for $0.3 \mathrm{~kg} \times$ yr exposure is a half-life limit on the $0 v 2 \beta$ decay of ${ }^{100} \mathrm{Mo}$ of $\mathrm{T}_{1 / 2}>9.5 \times 10^{22} \mathrm{yr}$. For this measurement, the background level in the ROI was 0.55 counts $/ \mathrm{keV} / \mathrm{kg} / \mathrm{yr}$ [65].

AMoRE-pilot measurement has demonstrated efficient $\alpha$ identification not only through the light yield, but also using high time resolution of MMCs, it was possible to separate the $\alpha$ band just by the rise time (see Figure 9). It is remarkable that the speed of the thermal response of MMCs (below $1 \mathrm{~ms}$ to be compared with $\sim 10 \mathrm{~ms}$ in NTDs) makes the background contribution due to random coincidences (Section 6.4) of $2 v 2 \beta$ events negligible. As a trade-off, this technique seems to provide an energy resolution about twice worse than NTDs.

Currently, ${ }^{48 d e p l} \mathrm{Ca}^{100} \mathrm{MoO}_{4}, \mathrm{Li}_{2}{ }^{100} \mathrm{MoO}_{4}, \mathrm{~Pb}^{100} \mathrm{MoO}_{4}$, and $\mathrm{Na}_{2}{ }^{100} \mathrm{Mo}_{2} \mathrm{O}_{7}$ are being considered as detector candidates for the AMoRE-II. The ${ }^{48 d e p l} \mathrm{Ca}^{100} \mathrm{MoO}_{4}(\mathrm{CMO})$ crystals 
used in AMoRE-Pilot and -I are promising candidates with high light yields but have several disadvantages, especially the required depletion of ${ }^{48} \mathrm{Ca}$ (since ${ }^{48} \mathrm{Ca}$ undergoes $2 \nu 2 \beta$ decay with $Q_{2 \beta}=4272 \mathrm{keV}$ and half-life $\mathrm{T}_{1 / 2}^{2 v}=4.2 \times 10^{19} \mathrm{yr}$, this isotope would introduce irreducible background in the ROI. Thus, at least factor 100 concentration reduction is required [66]). A high melting temperature makes the production cost comparatively high for a larger-scale experiment such as AMoRE-II. In addition, they show a high internal contamination, especially from the ${ }^{238} \mathrm{U}$ chain. AMoRE-I will compare ${ }^{48 d e p l} \mathrm{Ca}^{100} \mathrm{MoO}_{4}$ and $\mathrm{Li}_{2}{ }^{100} \mathrm{MoO}_{4}$ and choose the best candidate for the final phase of the experiment. The current baseline candidate for AMoRE-II is $\mathrm{Li}_{2}{ }^{100} \mathrm{MoO}_{4}$.
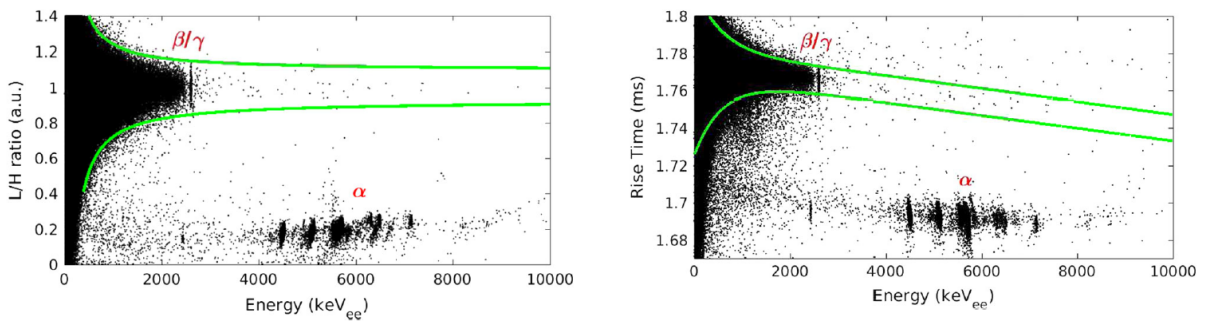

Figure 9. Scatter plot of the light yield (left) and the rise time (right) versus energy deposited in the phonon channel for one ${ }^{48 d e p l} \mathrm{Ca}^{100} \mathrm{MoO}_{4}$ crystal. The $\gamma / \beta$ band and the $\alpha$ clusters are clearly separated, as labeled in the figure. The figure is reprinted with permission from [65]. Creative Commons License CC BY 4.0.

AMoRE-II will be hosted in new cryogenic facility, installed at YemiLab, Korea. The experiment is planned to start in 2022, and it will be aiming at reaching the effective Majorana neutrino mass sensitivity down to the level of the inverted hierarchy of neutrino mass (13-25 meV) [67].

\subsection{4. ${ }^{48} \mathrm{Ca}: \mathrm{CaF}_{2}$ Bolometers}

${ }^{48} \mathrm{Ca}$ is an interesting isotope for $0 \nu 2 \beta$ decay searches, as it has very high transition energy $-4263 \mathrm{keV}$, above the background limit of natural $\beta$ decays from the ${ }^{238} \mathrm{U}$ chain $(3270 \mathrm{keV})$. The compound $\mathrm{CaF}_{2}$ was studied in the nineties of the last century by the Milano group, showing for the first time that $\alpha / \beta$ separation can be achieved with scintillating bolometers [68].

The CANDLES experiment is using $\mathrm{CaF}_{2}$ crystals as scintillators, and recently deployed a strategy for the development of $\mathrm{CaF}_{2}$ bolometric detectors. Bolometric measurements both with doped $\mathrm{CaF}_{2}(\mathrm{Eu})$ [69] and pure $\mathrm{CaF}_{2}$ crystals [28] were performed. The results obtained show a very good discrimination between $\gamma(\beta) / \alpha$ bands (see Figure 10), above $5 \sigma$ in both cases.
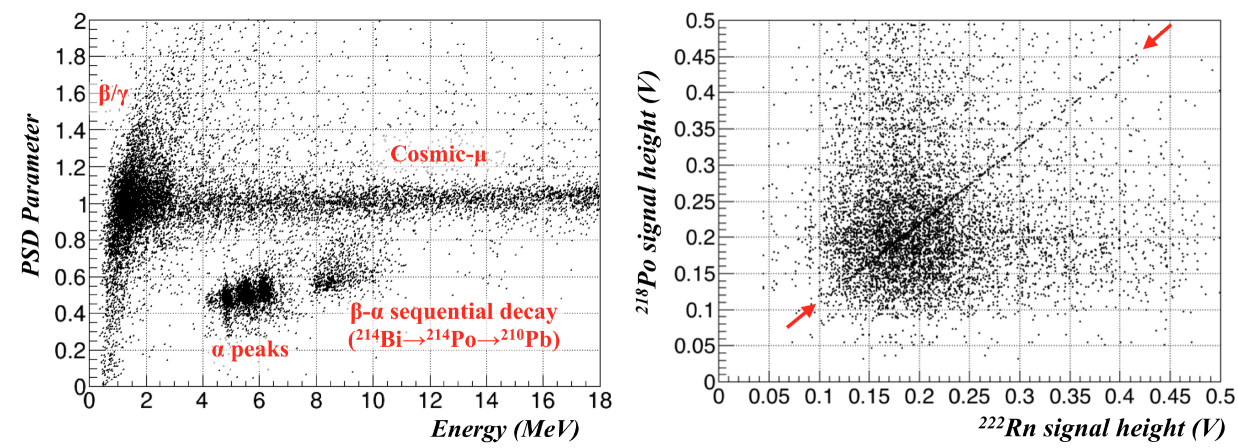

Figure 10. Left: scatter plot of energy, extracted from heat channel vs combined pulse shape discrimination (PSD) parameter: it includes light/heat energy ratio and light signals rise time. Right: demonstration of energy-position dependence through ${ }^{222} \mathrm{Rn}$ and ${ }^{218}$ Po decays. A strong correlation is observed. The figure is reprinted with permission from [28]. Creative Commons License CC BY 4.0. 
Both compounds types (doped and pure) are showing a strong position dependence in the heat channel, as shown in Figure 10 for $\mathrm{CaF}_{2}$ (pure) case. After the correction of such a correlation, an order of magnitude improvement is obtained for the energy resolution at $5 \mathrm{MeV}$ : from $1.8 \%$ to $0.18 \%$.

The group now is working on the preparation of a low-background cryogenic facility for future measurements of $\mathrm{CaF}_{2}$ scintillating bolometers [70].

\subsection{5. ${ }^{116} \mathrm{Cd}$ Bolometers: $\mathrm{R} \& \mathrm{D}$}

The advantages of the isotope ${ }^{116} \mathrm{Cd}$ is the high $\mathrm{Q}$-value and a reasonable isotopic abundance, with possible enrichment by gas centrifuges. Several experimental tests used $\mathrm{CdWO}_{4}$ crystals as scintillators for double beta decay searches. R\&D measurements of two massive cadmium tungstate crystals enriched in ${ }^{116} \mathrm{Cd}(82 \%)$ as scintillating bolometers were performed at the Modane and Canfranc underground laboratories.

The performance that was obtained in these measurements is satisfactory: $11-16 \mathrm{keV}$ FWHM energy resolution at $2615 \mathrm{keV}$ was demonstrated, along with a very efficient $\alpha$ discrimination thanks to the high light yield. However, the U/Th contamination has to be improved, if this compound has to be considered for a big-mass experiment. In addition to the traces of ${ }^{232} \mathrm{Th}$ and ${ }^{235} \mathrm{U}$ (ten(s) of $\mu \mathrm{Bq} / \mathrm{kg}$ ) and ${ }^{238} \mathrm{U}$ (hundreds of $\mu \mathrm{Bq} / \mathrm{kg}$ ), the presence of ${ }^{241} \mathrm{Am}$ and ${ }^{244} \mathrm{Cm}$ was detected, with combined activities of $0.14(1)$ and $0.23(1) \mathrm{mBq} / \mathrm{kg}$, respectively [71].

These results show that the ${ }^{116} \mathrm{CdWO}_{4}$ scintillating bolometer technology could be used for future $0 v 2 \beta$ searches in $\mathrm{Cd}$. However, the radiopurity of these crystals has to be improved before a deployment of this technology for large-scale experiments.

\subsubsection{Cherenkov Light Tagging: Neganov-Luke Enhanced Bolometers}

The case of ${ }^{130} \mathrm{Te}$ is peculiar, as this isotope has a uniquely high natural abundance ( $\sim 34 \%)$, which gives a significant advantage for costs of enrichment for a big scale experiment. In addition, sensitive experiments with natural isotopic composition can be considered if the total mass is high enough and the background index conveniently low.

CUORE demonstrated the viability of $\mathrm{TeO}_{2}$ bolometers, but any particle ID was absent. $\mathrm{TeO}_{2}$ crystals do not scintillate appreciably, so it was proposed to use Cherenkov light to tag $\alpha$ events: as $\beta$ s have a Cherenkov threshold at $50 \mathrm{keV}$, and $\alpha$ s at $400 \mathrm{MeV}$, the mere detection of light emission will reject $\alpha$ events. However, the detection of Cherenkov light can be challenging and requires low-threshold and high-sensitivity light detectors. The total amount of energy deposited in a light detector by Cherenkov photons is at least an order of magnitude lower than scintillation for all the scintillating compounds studied so far. To boost the performance of light detectors, the Neganov-Luke mode is used. Ge wafers are provided with Al electrodes, that boost the signals with a high voltage applied. In fact, the potential energy of the electron-hole pairs created by an ionizing event converts to heat during the drift to the electrodes.

The efficiency of double-readout Neganov-Luke-assisted bolometers was demonstrated in several tests with natural and enriched ${ }^{130} \mathrm{TeO}_{2}$ crystals [72-74].

\subsubsection{Future: CUPID Experiment}

The CUPID experiment (CUORE Upgrade with Particle IDentification) has set the limit on effective Majorana mass at $\sim 10 \mathrm{meV}$ as its main physics goal. CUPID-0 and CUPID-Mo demonstrators have shown good perspectives of the scintillating-bolometer technology, combining high energy resolution with efficient background rejection. The compound chosen for CUPID is $\mathrm{Li}_{2}{ }^{100} \mathrm{MoO}_{4}$, as ${ }^{100} \mathrm{Mo}$ offers several significant advantages:

- The calculations of nuclear matrix elements show that ${ }^{100}$ Mo may have one of the shortest half-lives among $0 v 2 \beta$ candidates [75];

- It is possible to have a large scale enrichment thanks to gas-centrifuge enrichment;

- $\quad$ The CUPID-Mo experiment demonstrated the high performance of scintillating $\mathrm{Li}_{2}{ }^{100} \mathrm{MoO}_{4}$ bolometers, as well as radiopurity, efficient $\alpha$ rejection, reproducibility and stability. 
In its baseline design, CUPID will consist of an array of about 1600 scintillating bolometers composed of $\mathrm{Li}_{2}{ }^{100} \mathrm{MoO}_{4}$ crystals weighing $280 \mathrm{~g}$ each-for a total ${ }^{100}$ Mo mass of about $250 \mathrm{~kg}$-coupled to germanium wafers acting as light detectors. The CUPID experiment is now finalizing its design [76]. Several measurements on smaller scale are ongoing to confirm the required performance [77].

A possible extension/upgrade of the CUPID experiment is being discussed already now, in case a further investigation of lower values of the effective Majorana neutrino mass is needed. The two possibly higher sensitivity phases are called CUPID-reach and CUPID-1T. The latter is an upgrade, that requires several cryostats to be built, to have in total 1 ton of ${ }^{100}$ Mo measured at the same time. CUPID-reach is a possible improvement of the current baseline configuration with additional background reduction- to reach a background index of $2 \times 10^{-5}$ counts $/ \mathrm{keV} / \mathrm{kg} / \mathrm{yr}$ [78].

\subsection{Surface $\beta$ Background}

The materials surrounding the absorber are usually highly radiopure, but when we are speaking about background-index levels of $10^{-4}$ counts $/ \mathrm{keV} / \mathrm{kg} / \mathrm{yr}$, contributions even at the tiniest level should be taken into account. According to the CUORE background model and evaluation of the background for CUPID, surface contamination can be limiting the sensitivity of an experiment even with efficient $\alpha$ rejection, if high sensitivities to the effective Majorana mass are required, down to the exploration of the normal ordering of neutrino masses.

We provide here examples of $\beta$ decays that are harmless in the crystal bulk (because of simultaneous or delayed $\alpha$ detection leading to discrimination), but are potentially contaminating the ROI in case of surface events generated in materials facing the detectors, as the $\alpha$ energy can be fully deposited outside the detector:

$$
\begin{aligned}
& { }^{238} \mathrm{U} \text { chain }::^{214} \mathrm{Bi} \stackrel{\beta}{\rightarrow}{ }^{214} \mathrm{Po} Q_{\beta}=3.3 \mathrm{MeV} \\
& { }^{232} \mathrm{Th} \text { chain }:{ }^{208} \mathrm{Tl} \stackrel{\beta}{\rightarrow}{ }^{208} \mathrm{~Pb} \quad Q_{\beta}=5.0 \mathrm{MeV}
\end{aligned}
$$

CUPID-0 demonstrated the capability to reject surface events through $\alpha-\alpha$ delayed coincidences [79], however, not at a level sufficient to reach background indices lower than $10^{-4}$ counts $/ \mathrm{keV} / \mathrm{kg} / \mathrm{y}$. Special technologies must be developed for that.

Before moving to the description to the only active method devised so far to reject surface $\beta$ events in double-beta-decay bolometers (CROSS approach, described in the next subsection), we remark that this background component can be mitigated in scintillating bolometers by a geometrical rearrangement of the main detector components (crystals, light detectors and copper holders) so that the amount of inactive material facing the main crystals is reduced by at least an order of magnitude with respect to the current configuration. In particular, the light detector can be used as an active shield against the $\beta$ particles emitted by the holder contamination. This is the approach followed by the BINGO project (Section 6.1.1).

\subsubsection{Surface $\beta$ Rejection: CROSS Project}

The CROSS (Cryogenic Rare-event Observatory with Surface Sensitivity) experiment proposes a new technology for background discrimination: active surface background rejection using bolometric detectors with thin metallic films. Such a film on the detector surface affects the conversion of energy deposited by particle interaction. The phonon absorption in the film leads to modification of the pulse shape for close-to-film events. Currently, the capability of surface/bulk discrimination both for $\alpha$ and $\beta$ particles was demonstrated in small $\left(20 \times 20 \times 10 \mathrm{~mm}^{3}\right) \mathrm{Li}_{2} \mathrm{MoO}_{4}$ and $\mathrm{TeO}_{2}$ crystals, measured in the cryogenic facility at IJCLab, Orsay. The R\&D included tests on several materials combinations (aluminum, palladium, aluminum-palladium bilayer) and various thicknesses, 
concluding with detector that demonstrated efficient surface event discrimination with Pd-Al bilayer, as one can see in Figure 11 [80].
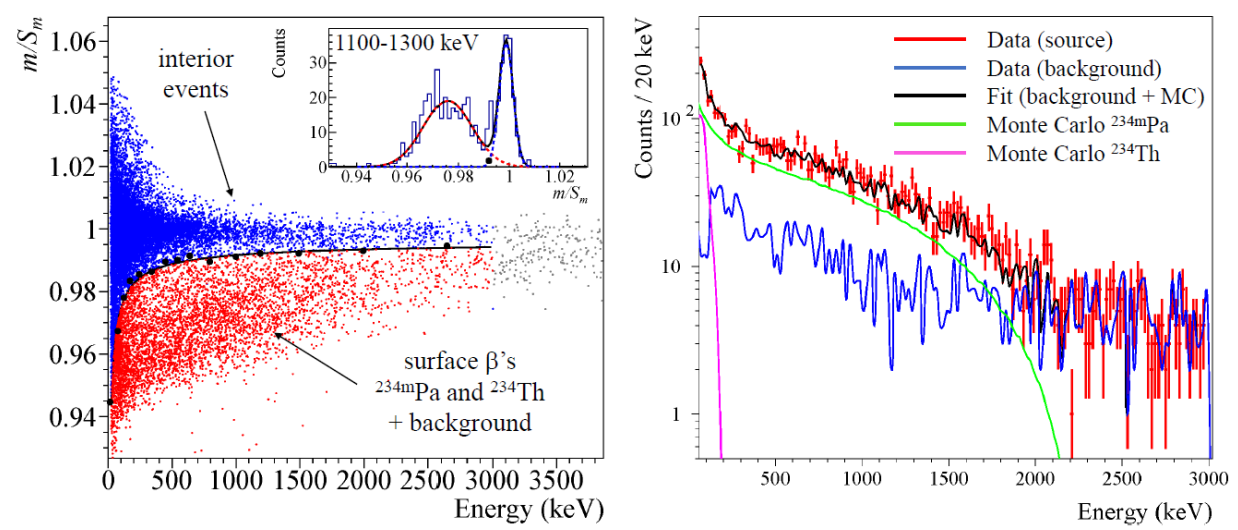

Figure 11. Particle identification obtained by a $\mathrm{Li}_{2} \mathrm{MoO}_{4}$ detector with an Al-Pd coating on one side, exposed to a uranium source. The $\alpha$ events are removed by a light-yield cut. Left: In a plot of the pulse-shape parameter versus energy, the surface events (in red) are separated by black line defining a $3 \sigma$ acceptance region for the bulk events (in blue). Right: Energy spectra (with and without source) of the selected surface events. The energy spectra with source is fitted with two simulated $\beta$ contributions of the uranium source and that of the background. The figure is reprinted with permission from [80]. Creative Commons License CC BY 4.0.

The concept is still to be proven on a bigger scale, and can be easily adopted by CUPID-like experiments for further phases of the project, and studies with ${ }^{130} \mathrm{TeO}_{2}$ and particle identification will renew. A mid-scale demonstrator with proposed technology is planned to be installed at the Canfranc underground laboratory in the next few years [81].

\section{4. $2 v 2 \beta$ Decay}

There is only an irreducible source of background for all the experiments-the $2 v-$ mode of double beta decay. Since the event signature is also two-electron emission, a $0 v 2 \beta$ event is indistinguishable from a $0 v 2 \beta$ one in terms of particle identification and event topology. The only tool for the control of this background is the accurate measurement of the total deposited energy.

An approach to mitigate this background could be the study of isotopes that have a long half-life of $2 v 2 \beta$ decay. However, this may not represent a solution, depending on the required sensitivity to observe $0 v 2 \beta$ decay. The ratio of the $0 v$ (as signal) and $2 v$ (as background) depends on the isotope only through its $2 v 2 \beta$ rate [82]:

$$
\frac{S}{B} \propto\left(\frac{Q_{2 \beta}}{\Delta E}\right)^{6} \frac{T_{1 / 2}^{2 v}}{T_{1 / 2}^{0 v}}
$$

This ratio clearly demonstrates the importance of high energy resolution $\Delta E$, especially for isotopes with short $2 v$-mode half-lives. In general, the energy resolution ensured by the bolometric approach is high enough to make this contribution negligible for all the studied isotopes, even for ${ }^{100} \mathrm{Mo}$ that has the shortest half-life among all the double-beta decay candidates $\left(T_{1 / 2}^{2 v}=7.12 \times 10^{18} \mathrm{yr}\right)$ [63].

There is, however, another possible source of background, related to random coincidences of $2 v 2 \beta$ events. In this case, the energy deposited by the two events sums up and produces an energy spectrum that ends up at $2 \times Q_{2 \beta}$ and is significantly populated in the ROI [83]. This contribution is particularly severe in CUPID, which combines the NTD technology (slow response) with ${ }^{100} \mathrm{Mo}$ (fast $2 v$ mode). That is why significant efforts are directed towards novel methods of rejection of pile-up events, that may be coming from $2 v 2 \beta$ decays pile-up within itself or pile-up with other background sources. Such methods 
allow to optimize event selection and improve the background in the ROI [84]. The preliminary tests and studies show that background indices down to $10^{-4}$ counts $/ \mathrm{keV} / \mathrm{kg} / \mathrm{yr}$ are reachable, compatible of the CUPID goal. Lower values demand probably faster readout or better light-detector time resolutions $[85,86]$.

\section{Conclusions}

The direct observation of a lepton charge violating process would enlarge greatly our understanding of the fundamentals of elementary particle theory and allow us to define the neutrino nature. This may lead to a progress in the theory of flavor and of the evolution of the Universe, including the origins of matter-antimatter asymmetry. Searches for the $0 \nu 2 \beta$ decay process have constantly progressed over the years starting from 1960s, reaching limits higher than $10^{26}$ yr. Bolometric $0 v 2 \beta$ experiments, which have started in around 1990, are showing now the best results on sensitivities on the effective Majorana neutrino mass when considering the exposure, thanks to high energy resolutions and efficiency. These properties also allow to perform measurements of the $2 v 2 \beta$ decay with very high precision. These measurements can be used for a better evaluation of the nuclear matrix elements for $0 v 2 \beta$ decay. With exposures of $\sim 10 \mathrm{~kg} \times$ year and background index at the level of $\sim 10^{-3}$ counts $/ \mathrm{keV} / \mathrm{kg} / \mathrm{yr}$, mid-scale demonstrators have reached $\sim 10^{24} \mathrm{yr}$ half-life limits. This advantage boosts interest in scaling up the mass of bolometric experiments. Both CUPID and AMoRE projects have set the goal of coverage of the inverted neutrino mass ordering region, reaching sensitivities to the effective Majorana mass at the level of 10-25 meV. Both experiments have chosen the ${ }^{100}$ Mo isotope, due to favorable predictions on the half-life and its possible embedding in highly-performing bolometers. The required sensitivity for the inverted hierarchy milestone would be at the level of $\sim 10^{27} \mathrm{yr}$. In addition, several projects, proposing innovative techniques, are already preparing the way for a further extension of the sensitivity, if it is required, as well as mature technologies for the exploration of other $0 v 2 \beta$ isotopes-if the discovery occurs, it should be confirmed in several nuclei.

Funding: This research received no external funding.

Conflicts of Interest: The author declares no conflict of interest.

\section{References}

1. Iachello, F. Open problems in neutrino physics. J. Phys. 2018, 1056, 012027. [CrossRef]

2. Davis, R.; Harmer, D.S.; Hoffman, K.C. Search for Neutrinos from the Sun. Phys. Rev. Lett. 1968, 20, 1205-1209. [CrossRef]

3. Tretyak, V.I.; Zdesenko, Y.G. Tables of double beta decay data-An update. At. Data Nucl. Data Tables 2002, 80, 83-116. [CrossRef]

4. Barabash, A. Precise Half-Life Values for Two-Neutrino Double- $\beta$ Decay: 2020 Review. Universe 2020, 6, 159. [CrossRef]

5. Adams, D.Q.; Alduino, C.; Alfonso, K.; Avignone, F.T.; Azzolini, O.; Bari, G.; Bellini, F.; Benato, G.; Biassoni, M.; Branca, A.; et al. Measurement of the $2 v \beta \beta$ Decay Half-Life of ${ }^{130}$ Te with CUORE. Phys. Rev. Lett. 2021, 126, 171801. [CrossRef] [PubMed]

6. Furry, W.H. On Transition Probabilities in Double Beta Desintegration. Phys. Rev. 1939, 56, 1184-1193. [CrossRef]

7. Stoica, S.; Mirea, M. Phase Space Factors for Double-Beta Decays. Front. Phys. 2019, 7, 12. [CrossRef]

8. Ejiri, H. Nuclear Matrix Elements for $\beta$ and $\beta \beta$ Decays and Quenching of the Weak Coupling $g_{A}$ in QRPA. Front. Phys. 2019, 7, 30. [CrossRef]

9. Barea, J.; Kotila, J.; Iachello, F. $0 v \beta \beta$ and $2 v \beta \beta$ nuclear matrix elements in the interacting boson model with isospin restoration. Phys. Rev. C 2015, 91, 034304. [CrossRef]

10. Zyla, P.A.; Barnett, R.M.; Beringer, J.; Dahl, O.; Dwyer, D.A.; Groom, D.E.; Lin, C.-J.; Lugovsky, K.S.; Pianori, E.; Robinson, D.J.; et al. Review of Particle Physics:Neutrino Masses, Mixing, and Oscillations. Prog. Theor. Exp. Phys. 2020, 2020, 083C01. [CrossRef]

11. Esteban, I.; Gonzalez-Garcia, M.C.; Maltoni, M.; Schwetz, T.; Zhou, A. The fate of hints: Updated global analysis of three-flavor neutrino oscillations. J. High Energy Phys. 2020, 2020, 178. [CrossRef]

12. NuFIT 5.0. 2020. Available online: www.nu-fit.org (accessed on 30 October 2021).

13. Gando, A.; Gando, Y.; Hachiya, T.; Hayashi, A.; Hayashida, S.; Ikeda, H.; Inoue, K.; Ishidoshiro, K.; Karino, Y.; Koga, M.; et al. Search for Majorana Neutrinos Near the Inverted Mass Hierarchy Region with KamLAND-Zen. Phys. Rev. Lett. 2016, 117, 082503. [CrossRef]

14. Agostini, M.; Araujo, G.R.; Bakalyarov, A.M.; Balata, M.; Barabanov, I.; Baudis, L.; Bauer, C.; Bellotti, E.; Belogurov, S.; Bettini, A.; et al. Final Results of GERDA on the Search for Neutrinoless Double- $\beta$ Decay. Phys. Rev. Lett. 2020, 125, 252502. [CrossRef] 
15. Adams, D.Q.; Alduino, C.; Alfonso, K.; Avignone, F.T.; Azzolini, O.; Bari, G.; Bellini, F.; Benato, G.; Biassoni, M.; Branca, A.; et al. Improved Limit on Neutrinoless Double-Beta Decay in ${ }^{130}$ Te with CUORE. Phys. Rev. Lett. 2020, 124, 122501. [CrossRef]

16. Azzolini, O.; Beeman, J.W.; Bellini, F.; Beretta, M.; Biassoni, M.; Brofferio, C.; Bucci, C.; Capelli, S.; Cardani, L.; Carniti, P.; et al. Final Result of CUPID-0 Phase-I in the Search for the ${ }^{82}$ Se Neutrinoless Double- $\beta$ Decay. Phys. Rev. Lett. 2019, $123,032501$. [CrossRef]

17. Pagnanini, L.; On Behalf of the CUPID-0 Collaboration. The ultimate limit on ${ }^{82}$ Se neutrinoless double-beta decay search with CUPID-0. In Proceedings of the 17th International Conference on Topics in Astroparticle and Underground Physics (TAUP 2021), online, 26 August-6 September 2021.

18. Armengaud, E.; Augier, C.; Barabash, A.S.; Bellini, F.; Benato, G.; Benoît, A.; Beretta, M.; Bergé, L.; Billard, J.; Borovlev, Y.A.; et al. New Limit for Neutrinoless Double-Beta Decay of ${ }^{100}$ Mo from the CUPID-Mo Experiment. Phys. Rev. Lett. 2021, 126, 181802. [CrossRef]

19. Welliver, B.; On Behalf of the CUPID-Mo Collaboration. New results from the CUPID-Mo demonstrator on the ${ }^{100}$ Mo $0 v \beta \beta$ decay half-life. In Proceedings of the 17th International Conference on Topics in Astroparticle and Underground Physics (TAUP 2021), online, 26 August-6 September 2021.

20. Arnold, R.; Augier, C.; Baker, J.D.; Barabash, A.S.; Basharina-Freshville, A.; Blondel, S.; Blot, S.; Bongrand, M.; Brudanin, V.; Busto, J.; et al. Measurement of the $2 v \beta \beta$ decay half-life of ${ }^{150} \mathrm{Nd}$ and a search for $0 v \beta \beta$ decay processes with the full exposure from the NEMO-3 detector. Phys. Rev. D 2016, 94, 072003. [CrossRef]

21. Arnold, R.; Augier, C.; Baker, J.D.; Barabash, A.S.; Basharina-Freshville, A.; Blondel, S.; Blot, S.; Bongrand, M.; Boursette, D.; Brudanin, V.; et al. Measurement of the $2 v \beta \beta$ decay half-life and search for the $0 v \beta \beta$ decay of ${ }^{116} C d$ with the NEMO-3 detector. Phys. Rev. D 2017, 95, 012007. [CrossRef]

22. Arnold, R.; Augier, C.; Baker, J.D.; Barabash, A.S.; Basharina-Freshville, A.; Blondel, S.; Blot, S.; Bongrand, M.; Brudanin, V.; Busto, J.; et al. Results of the search for neutrinoless double- $\beta$ decay in ${ }^{100}$ Mo with the NEMO-3 experiment. Phys. Rev. D 2015, 92, 072011. [CrossRef]

23. Arnold, R.; Augier, C.; Bakalyarov, A.M.; Baker, J.D.; Barabash, A.S.; Basharina-Freshville, A.; Blondel, S.; Blot, S.; Bongrand, M.; Brudanin, V.; et al. Measurement of the double-beta decay half-life and search for the neutrinoless double-beta decay of ${ }^{48} \mathrm{Ca}$ with the NEMO-3 detector. Phys. Rev. D 2016, 93, 112008. [CrossRef]

24. Barabash, A.S.; Basharina-Freshville, A.; Blot, S.; Bongrand, M.; Bourgeois, C.; Breton, D.; Brudanin, V.; Burešovà, H.; Busto, J.; Caffrey, A.J.; et al. Calorimeter development for the SuperNEMO double beta decay experiment. Nucl. Instrum. Methods Phys. Res. Sect. A Accel. Spectr. Detect. Assoc. Equip. 2017, 868, 98-108. [CrossRef]

25. Biassoni, M.; Cremonesi, O. Search for neutrino-less double beta decay with thermal detectors. Prog. Part. Nucl. Phys. 2020, 114, 103803. [CrossRef]

26. Giuliani, A.; Cadenas, J.J.G.; Pascoli, S.; Previtali, E.; Saakyan, R.; Schaeffner, K.; Schoenert, S. Double Beta Decay APPEC Committee Report. arXiv 2020, arXiv:hep-ex/1910.04688.

27. Artusa, D.R.; Avignone, F.T.; Azzolini, O.; Balata, M.; Banks, T.I.; Bari, G.; Beeman, J.; Bellini, F.; Bersani, A.; Biassoni, M.; et al. Exploring the neutrinoless double beta decay in the inverted neutrino hierarchy with bolometric detectors. Eur. Phys. J. C 2014, 74, 3096. [CrossRef]

28. Tetsuno, K.; Ajimura, S.; Akutagawa, K.; Chan, W.M.; Fushimi, K.; Hazama, R.; Iida, T.; Ikeyama, Y.; Khai, B.T.; Kishimoto, T.; et al. Status of ${ }^{48}$ Ca double beta decay search and its future prospect in CANDLES. J. Phys. Conf. Ser. 2020, 1268, 012132. [CrossRef]

29. Argyriades, J.; Arnold, R.; Augier, C.; Baker, J.; Barabash, A.S.; Basharina-Freshville, A.; Bongrand, M.; Broudin-Bay, G.; Brudanin, V.; Caffrey, A.J.; et al. Measurement of the two neutrino double beta decay half-life of Zr-96 with the NEMO-3 detector. Nucl. Phys. A 2010, 847, 168-179. [CrossRef]

30. Danevich, F.A.; Barabash, A.S.; Belli, P.; Bernabei, R.; Cappella, F.; Caracciolo, V.; Cerulli, R.; Chernyak, D.M.; d'Angelo, S.; Incicchitti, A.; et al. Search for double beta decay of ${ }^{116} \mathrm{Cd}$ with enriched ${ }^{116} \mathrm{CdWO}_{4}$ crystal scintillators (Aurora experiment). J. Phys. Conf. Ser. 2016, 718, 062009. [CrossRef]

31. Turkevich, A.L.; Economou, T.E.; Cowan, G.A. Double beta decay of U-238. Phys. Rev. Lett. 1991, 67, 3211-3214. [CrossRef]

32. Giuliani, A. Particle and radiation detection with low-temperature devices. Phys. B Condens. Matter 2000, 280, 501-508. [CrossRef]

33. Pirro, S.; Mauskopf, P. Advances in Bolometer Technology for Fundamental Physics. Annu. Rev. Nucl. Part. Sci. 2017, 67, 161-181. [CrossRef]

34. Wang, N.; Wellstood, F.C.; Sadoulet, B.; Haller, E.E.; Beeman, J. Electrical and thermal properties of neutron-transmutation-doped Ge at $20 \mathrm{mK}$. Phys. Rev. B 1990, 41, 3761-3768. [CrossRef] [PubMed]

35. Appel, J.W.; Galeazzi, M. Two models for bolometer and microcalorimeter detectors with complex thermal architectures. Nucl. Instrum. Methods Phys. Res. Sect. A 2006, 562, 272-280. [CrossRef]

36. Bandler, S.R.; Enss, C.; Lanou, R.E.; Maris, H.J.; More, T.; Porter, F.S.; Seidel, G.M. Metallic magnetic bolometers for particle detection. J. Low Temp. Phys. 1993, 93, 709-714. [CrossRef]

37. Lee, C.; Jo, H.; Kang, C.; Kim, G.B.; Kim, I.; Kim, Y.; Lee, H.J.; So, J. Vibration Mitigation for a Cryogen-Free Dilution Refrigerator for the AMoRE-Pilot Experiment. J. Low Temp. Phys. 2018, 193, 786-792. [CrossRef]

38. Maisonobe, R.; Billard, J.; De Jesus, M.; Dumoulin, L.; Juillard, A.; Marnieros, S.; Misiak, D.; Sayah, S.; Vagneron, L. Experimental study and modeling cryogenic detectors decoupling within dry cryostat. J. Low Temp. Phys. 2017, 193, 819-826. [CrossRef] 
39. Alduino, C.; Alessandria, F.; Balata, M.; Biare, D.; Biassoni, M.; Bucci, C.; Caminata, A.; Canonica, L.; Cappelli, L.; Ceruti, G.; et al. The CUORE cryostat: An infrastructure for rare event searches at milliKelvin temperatures. Cryogenics 2019, 102, 9-21. [CrossRef]

40. Singh, M.; Sharma, V.; Kumar, A.; Pandey, A.; Singh, V.; Wong, H.T. Required sensitivity to search the neutrinoless double beta decay in ${ }^{124}$ Sn. Indian J. Phys. 2018, 94,1-13. [CrossRef]

41. Bacrania, M.K.; Hoover, A.S.; Karpius, P.J.; Rabin, M.W.; Rudy, C.R.; Vo, D.T.; Beall, J.A.; Bennett, D.A.; Doriese, W.B.; Hiltonm G.C.; et al. Large-Area Microcalorimeter Detectors for Ultra-High-Resolution X-Ray and Gamma-Ray Spectroscopy. IEEE Trans. Nucl. Sci. 2009, 56, 2299-2302. [CrossRef]

42. Nanal, V. Search for neutrinoless double beta decay in ${ }^{124}$ Sn. EPJ Web Conf. 2014, 66, 08005. [CrossRef]

43. Mazumdar, A.; Garai, A.; Krishnamoorthy, H.; Gupta, G.; Reza, A.; Thamizhavel, A.; Nanal, V.; Pillay, R.G.; Shrivastava, A. Studies on $\beta \rightleftharpoons \alpha$ transition in Sn and Sn-rich alloys for a cryogenic tin bolometer. Mater. Res. Express 2019. 6, 076521. [CrossRef]

44. Andreotti, E.; Arnaboldi, C.; Avignone, F.T.; Balata, M.; Bandac, I.; Barucci, M.; Beeman, J.W.; Bellini, F.; Brofferio, C.; Bryant, A.; et al. ${ }^{130}$ Te neutrinoless double-beta decay with CUORICINO. Astropart. Phys. 2011, 34, 822-831. [CrossRef]

45. Alfonso, K.; Artusa, D.R.; Avignone, F.T.; Azzolini, O.; Balata, M.; Banks, T.I.; Bari, G.; Beeman, J.W.; Bellini, F.; Bersani, A.; et al. Search for Neutrinoless Double-Beta Decay of ${ }^{130}$ Te with CUORE-0. Phys. Rev. Lett. 2015, 115, 102502. [CrossRef]

46. Adams, D.Q.; Alduino, C.; Alessandria, F.; Alfonso, K.; Andreotti, E.; Avignone, F.T.; Azzolini, O.; Balata, M.; Bandac, I.; Banks, T.I.; et al. CUORE Opens the Door to Tonne-scale Cryogenics Experiments. Prog. Part. Nucl. Phys. 2021, 103902. [CrossRef]

47. Alduino, C.; Alfonso, K.; Artusa, D.R.; Avignone, F.T.; Azzolini, O.; Banks, T.I.; Bari, G.; Beeman, J.W.; Bellini, F.; Benato, G.; et al. The projected background for the CUORE experiment. Eur. Phys. J. C 2017, 77, 543. [CrossRef]

48. Singh, M.K.; Wong, H.T.; Singh, L.; Sharma, V.; Singh, V.; Yue, Q. Exposure-background duality in the searches of neutrinoless double beta decay. Phys. Rev. D 2020, 101, 013006. [CrossRef]

49. Poda, D.; Giuliani, A. Low background techniques in bolometers for double-beta decay search. Int. Natl. J. (Wash.) Mod. Phys. A 2017, 32, 1743012. [CrossRef]

50. Nones, C.; On Behalf of the BINGO Collaboration. BINGO: Bi-Isotope $0 v 2 \beta$ Next Generation Observatory. In Proceedings of the 17th International Conference on Topics in Astroparticle and Underground Physics (TAUP 2021), online, 26 August-6 September 2021.

51. Danevich, F. Radiopure tungstate and molybdate crystal scintillators for double beta decay experiments. Int. J. Mod. Phys. A 2017, 32, 17430084. [CrossRef]

52. Arnaboldi, C.; Brofferio, C.; Bryant, A.; Bucci, C.; Canonica, L.; Capelli, S.; Carrettoni, M.; Clemenza, M.; Dafinei, I.; Di Domizio, S.; et al. Production of high purity $\mathrm{TeO}_{2}$ single crystals for the study of neutrinoless double beta decay. J. Cryst. Growth 2010, 312, 2999-3008. [CrossRef]

53. Clemenza, M.; Maiano, C.; Pattavina, L.; Previtali, E. Radon-induced surface contaminations in low background experiments. Eur. Phys. J. C 2011, 71, 1805. [CrossRef]

54. Poda, D. Scintillation in Low-Temperature Particle Detectors. Physics 2021, 3, 473-535. [CrossRef]

55. Azzolini, O.; Beeman, J.W.; Bellini, F.; Beretta, M.; Biassoni, M.; Brofferio, C.; Bucci, C.; Capelli, S.; Cardani, L.; Carniti, P.; et al. Evidence of Single State Dominance in the Two-Neutrino Double- $\beta$ Decay of ${ }^{82}$ Se with CUPID-0. Phys. Rev. Lett. 2019, 123, 262501. [CrossRef] [PubMed]

56. Chiesa, D.; Azzolini, O.; Beeman, J.W.; Bellini, F.; Beretta, M.; Biassoni, M.; Brofferio, C.; Bucci, C.; Capelli, S.; Cardani, L.; et al. Double beta decay results from the CUPID-0 experiment. arXiv 2020, arXiv:2012.00644.

57. Pagnanini, L.; Azzolini, O.; Beeman, J.W.; Bellini, F.; Beretta, M.; Biassoni, M.; Brofferio, C.; Bucci, C.; Capelli, S.; Cardani, L.; et al. Results on ${ }^{82}$ Se $2 v \beta \beta$ with CUPID-0 Phase I. J. Phys. Conf. Ser. 2020, 1643, 012025. [CrossRef]

58. Azzolini, O.; Barrera, M.T.; Beeman, J.W.; Bellini, F.; Beretta, M.; Biassoni, M.; Brofferio, C.; Bucci, C.; Canonica, L.; Capelli, S.; et al. First Result on the Neutrinoless Double- $\beta$ Decay of ${ }^{82}$ Se with CUPID-0. Phys. Rev. Lett. 2018, 120, 232502. [CrossRef] [PubMed]

59. Azzolini, O.; Beeman, J.W.; Bellini, F.; Beretta, M.; Biassoni, M.; Brofferio, C.; Bucci, C.; Capelli, S.; Cardani, L.; Carniti, P.; et al. First search for Lorentz violation in double beta decay with scintillating calorimeters. Phys. Rev. D 2019, 100, 092002. [CrossRef]

60. Azzolini, O.; Beeman, J.W.; Bellini, F.; Beretta, M.; Biassoni, M.; Brofferio, C.; Bucci, C.; Capelli, S.; Cardani, L.; Celi, E.; et al. Search for neutrinoless double beta decay of ${ }^{64} \mathrm{Zn}$ and ${ }^{70} \mathrm{Zn}$ with CUPID-0. Eur. Phys. J. C 2020, 80, 702. [CrossRef]

61. Armengaud, E.; Arnaud, Q.; Augier, C.; Benoît, A.; Bergé, L.; Bergmann, T.; Billard, J.; de Boissière, T.; Bres, G.; Broniatowski, A.; et al. Performance of the EDELWEISS-III experiment for direct dark matter searches. J. Instrum. 2017, 12, P08010. [CrossRef]

62. Armengaud, E.; Augier, C.; Barabash, A.S.; Bellini, F.; Benato, G.; Benoît, A.; Beretta, M.; Bergé, L.; Billard, J.; Borovlev, Y.A.; et al. The CUPID-Mo experiment for neutrinoless double-beta decay: Performance and prospects. Eur. Phys. J. C 2020, 80, 44 . [CrossRef]

63. Armengaud, E.; Augier, C.; Barabash, A.S.; Bellini, F.; Benato, G.; Benoît, A.; Beretta, M.; Bergé, L.; Billard, J.; Borovlev, Y.A.; et al. Precise measurement of $2 v \beta \beta$ decay of ${ }^{100}$ Mo with the CUPID-Mo detection technology. Eur. Phys. J. C 2020, 80, 1-11. [CrossRef]

64. Lee, M.H. AMoRE: A search for neutrinoless double-beta decay of ${ }^{100}$ Mo using low-temperature molybdenum-containing crystal detectors. J. Instrum. 2020, 15, C08010. [CrossRef]

65. Alenkov, V.; Bae, H.W.; Beyer, J.; Boiko, R.S.; Boonin, K.; Buzanov, O.; Chanthima, N.; Cheoun, M.K.; Chernyak, D.M.; Choe, J.S.; et al. First results from the AMoRE-Pilot neutrinoless double beta decay experiment. Eur. Phys. J. C 2019, 79, 791. [CrossRef] 
66. Alenkov, V.; Aryal, P.; Beyer, J.; Boiko, R.S.; Boonin, K.; Buzanov, O.; Chanthima, N.; Cheoun, M.K.; Chernyak, D.M.; Choi, J.; et al. Technical Design Report for the AMoRE 0v $\beta \beta$ Decay Search Experiment. arXiv 2015, arXiv:physics.ins-det/1512.05957.

67. Yoomin, O.; On Behalf of the AMoRe Collaboration. Status of AMoRE. In Proceedings of the 17th International Conference on Topics in Astroparticle and Underground Physics (TAUP 2021), online, 26 August-6 September 2021.

68. Alessandrello, A.; Bashkirov, V.; Brofferio, C.; Bucci, C.; Camin, D.V.; Cremonesi, O.; Fiorini, E.; Gervasio, G.; Giuliani, A.; Nucciotti, A.; et al. A scintillating bolometer for experiments on double beta decay. Phys. Lett. B 1998, 420, 109-113. [CrossRef]

69. Li, X.; Kwon, D.H.; Tetsuno, K.; Kim, I.; Kim, H.L.; Lee, H.J.; Yoshida, S.; Kim, Y.H.; Lee, M.K.; Umehara, S.; et al. Study of a Large $\mathrm{CaF}_{2}(\mathrm{Eu})$ Scintillating Bolometer for Neutrinoless Double Beta Decay. J. Phys. Conf. Ser. 2020, 1468, 012116. [CrossRef]

70. Minami, Y.; On Behalf of the CANDLES Collaboration. Status of ${ }^{48}$ Ca double beta decay search in CANDLES. In Proceedings of the 17th International Conference on Topics in Astroparticle and Underground Physics (TAUP 2021), online, 26 August-6 September 2021.

71. Helis, D.L.; Bandac, I.C.; Barabash, A.S.; Billard, J.; Chapellier, M.; de Combarieu, M.; Danevich, F.A.; Dumoulin, L.; Gascon, J.; Giuliani, A.; et al. Neutrinoless Double-Beta Decay Searches with Enriched ${ }^{116} \mathrm{CdWO}_{4}$ Scintillating Bolometers. J. Low Temp. Phys. 2019, 199, 467-474. [CrossRef]

72. Bergé, L.; Chapellier, M.; de Combarieu, M.; Dumoulin, L.; Giuliani, A.; Gros, M.; de Marcillac, P.; Marnieros, S.; Nones, C.; Novati, V.; et al. Complete event-by-event $\alpha / \gamma(\beta)$ separation in a full-size $\mathrm{TeO}_{2}$ CUORE bolometer by Neganov-Luke-magnified light detection. Phys. Rev. C 2018, 97, 1-5. [CrossRef]

73. Artusa, D.R.; Avignone, F.T.; Beeman, J.W.; Dafinei, I.; Dumoulin, L.; Ge, Z.; Giuliani, A.; Gotti, C.; de Marcillac, P.; Marnieros, S. Enriched $\mathrm{TeO}_{2}$ bolometers with active particle discrimination: Towards the CUPID experiment. Phys. Lett. B 2017, 767, 321-329. [CrossRef]

74. Casali, N.; Dumoulin, L.; Giuliani, A.; Mancuso, M.; de Marcillac, P.; Marnieros, S.; Nagorny, S.; Nones, C.; Olivieri, E.; Pagnanini, L.; et al. Background Suppression in Massive $\mathrm{TeO}_{2}$ Bolometers with Neganov-Luke Amplified Light Detectors. J. Low Temp. Phys. 2015, 184, 286-291. [CrossRef]

75. Engel, J.; Menéndez, J. Status and future of nuclear matrix elements for neutrinoless double-beta decay: A review. Rep. Prog. Phys. 2017, 80, 046301. [CrossRef]

76. The CUPID Interest Group. CUPID pre-CDR. arXiv 2019, arXiv:physics.ins-det/1907.09376.

77. Armatol, A.; Armengaud, E.; Armstrong, W.; Augier, C.; Avignone, F.T.; Azzolini, O.; Barabash, A.; Bari, G.; Barresi, A.; Baudin, D.; et al. Characterization of cubic $\mathrm{Li}_{2}^{100} \mathrm{MoO}_{4}$ crystals for the CUPID experiment. Eur. Phys. J. C 2020, 81, 104. [CrossRef]

78. Ouellet, J.; On Behalf of the CUPID Collaboration. CUPID: A Next-Generation Bolometric 0v $\beta \beta$ Decay Experiment. In Proceedings of the Presentation at 17th International Conference on Topics in Astroparticle and Underground Physics (TAUP 2021), online, 26 August-6 September 2021.

79. Azzolini, O.; Beeman, J.W.; Bellini, F.; Beretta, M.; Biassoni, M.; Brofferio, C.; Bucci, C.; Capelli, S.; Cardani, L.; Carniti, P.; et al. Background identification in cryogenic calorimeters through $\alpha-\alpha$ delayed coincidences. Eur. Phys. J. C 2021, 81, 722. [CrossRef] [PubMed]

80. Bandac, I.C.; Barabash, A.S.; Bergé, L.; Bourgeois, C.; Calvo-Mozota, J.M.; Carniti, P.; Chapellier, M.; de Combarieu, M.; Dafinei, I.; Danevich, F.A.; et al. Phonon-mediated crystal detectors with metallic film coating capable of rejecting $\alpha$ and $\beta$ events induced by surface radioactivity. Appl. Phys. Lett. 2021, 118, 184105. [CrossRef]

81. Bandac, I.C.; Barabash, A.S.; Bergé, L.; Bourgeois, C.; Calvo-Mozota, J.M.; Carniti, P.; Chapellier, M.; de Combarieu, M.; Dafinei, I.; Danevich, F.A.; et al. The 0v2 $\beta$-decay CROSS experiment: Preliminary results and prospects. J. High Energy Phys. 2020, 2020. [CrossRef]

82. Dolinski, M.J.; Poon, A.W.; Rodejohann, W. Neutrinoless Double-Beta Decay: Status and Prospects. Annu. Rev. Nucl. Part. Sci. 2019, 69, 219-251. [CrossRef]

83. Chernyak, D.M.; Danevich, F.A.; Giuliani, A.; Olivieri, E.; Tenconi, M.; Tretyak, V.I. Random coincidence of $2 v 2 \beta$ decay events as a background source in bolometric $0 v 2 \beta$ decay experiments. Eur. Phys. J. C 2012, 72, 1989. [CrossRef]

84. Armatol, A.; Armengaud, E.; Armstrong, W.; Augier, C.; Avignone, F.T.; Azzolini, O.; Barabash, A.; Bari, G.; Barresi, A.; Baudin, D.; et al. Novel technique for the study of pileup events in cryogenic bolometers. Phys. Rev. C 2021, 104, 015501. [CrossRef]

85. Chernyak, D.M.; Danevich, F.A.; Dumoulin, L.; Giuliani, A.; Mancuso, M.; de Marcillac, P.; Marnieros, S.; Nones, C.; Olivieri, E.; Poda, D.V.; et al. Rejection of randomly coinciding events in $\mathrm{Li}_{2}^{100} \mathrm{MoO}_{4}$ scintillating bolometers using light detectors based on the Neganov-Luke effect. Eur. Phys. J. C 2016, 77, 3. [CrossRef]

86. Casali, N.; Cardani, L.; Colantoni, I.; Cruciani, A.; Di Domizio, S.; Martinez, M.; Pettinari, G.; Vignati, M. Phonon and light read out of a $\mathrm{Li}_{2} \mathrm{MoO}_{4}$ crystal with multiplexed kinetic inductance detectors. Eur. Phys. J. C 2019, 79, 724. [CrossRef] 